TRANSACTIONS OF THE

AMERICAN MATHEMATICAL SOCIETY

Volume 355 , Number 10, Pages 4201-4222

S 0002-9947(03)03350-6

Article electronically published on June 10, 2003

\title{
SUB-BUNDLES OF THE COMPLEXIFIED TANGENT BUNDLE
}

\author{
HOWARD JACOBOWITZ AND GERARDO MENDOZA
}

\begin{abstract}
We study embeddings of complex vector bundles, especially line bundles, in the complexification of the tangent bundle of a manifold. The aim is to understand implications of properties of interest in partial differential equations.
\end{abstract}

\section{INTRODUCTION}

An involutive structure on a smooth $n$-manifold $\mathcal{M}$ is a smooth sub-bundle $\mathcal{V}$ of the complexified tangent bundle $\mathbb{C} T \mathcal{M}$ of $\mathcal{M}$ that satisfies

$$
[\mathcal{V}, \mathcal{V}] \subset \mathcal{V}
$$

that is, if $X$ and $Y$ are sections of $\mathcal{V}$, then their Lie bracket $[X, Y]$ is again a section of $\mathcal{V}$. Involutive structures give rise to complexes of differential operators, and from that point of view, they are well studied in partial differential equations, both locally and globally. It is thus natural to ask questions such as how rich the set of such vector sub-bundles is, and to what extent properties of interest in PDEs can be satisfied. For example, is every complex vector bundle $E \rightarrow \mathcal{M}$ of rank $\leq \operatorname{dim} \mathcal{M}$ realizable as a sub-bundle of $\mathbb{C} T \mathcal{M}$ ? Not generally (obviously), but yes if rank $E \leq(n+1) / 2$ (the stable case). If $E$ can be realized as a sub-bundle of $\mathbb{C} T \mathcal{M}$, can it be realized as an involutive sub-bundle? The answer to this is, not generally: there are topological conditions, due to Bott 3 and additional secondary conditions determined by Shulman [24] (see also 29]), that must be satisfied if $E$ can be realized as an involutive sub-bundle of $\mathbb{C} T \mathcal{M}$. For vector bundles of rank $\leq(n+1) / 2$, the conditions are vacuous. Other questions one may ask include whether a locally integrable structure (see Section [7 for the definition) admits a hypo-analytic structure (defined in Section [8) and which vector bundles can be embedded in $\mathbb{C} T \mathcal{M}$ so that they become hypo-complex.

This paper is a start on such studies. Its goal is to gain some understanding of complex line sub-bundles of the complexified tangent bundle (so involutivity comes for free) in connection with the PDEs they define. Involutivity together with properties of interest in PDEs or differential geometry may be very strong conditions on the manifold. This of course is illustrated by the fact that not all even-dimensional manifolds admit an almost complex structure (see, for example, 7], 10], [11, 27]), and those that do may not admit a complex structure (see [12). Other examples come from the fact that on any compact orientable 3-manifold, every complex rank

Received by the editors September 18, 2002 and, in revised form, February 27, 2003.

2000 Mathematics Subject Classification. Primary 57R22, 58J10; Secondary 35F05, 35N10.

Key words and phrases. Complexified tangent bundle, hypo-complex vector fields, Chern class, CR structure, characteristic points. 
two vector bundle with first Chern class in $2 H^{2}(\mathcal{M}, \mathbb{Z})$ embeds as a sub-bundle $\mathcal{V}$ with $\mathcal{V}+\overline{\mathcal{V}}=\mathbb{C} T \mathcal{M}$ (see Corollary [5.8). However, only for a specified list of 3-manifolds (see [5], [9]) can such a sub-bundle be involutive. These involutive structures are called elliptic and are equivalent to transversely holomorphic foliations. Note that every compact three-manifold admits an involutive real rank two bundle, since the manifold admits a codimension one foliation, Wood [32]. Thus in terms of the characteristic set of the sub-bundle we have two extremes: Every compact three-manifold admits a rank two involutive structure with the characteristic set equal to the manifold, but only a special class of three-manifolds admits a rank two involutive structure with the characteristic set empty.

We begin in Section 2 with a basic result about embeddings, and discuss briefly Bott's necessary condition for involutivity.

In Section 3 we consider the case of embeddings of complex line bundles $E \rightarrow \mathcal{M}$ as CR sub-bundles. This just means that one seeks to embed the underlying real bundle $E_{\mathbb{R}}$ in $T \mathcal{M}$. On this aspect of the problem, the work of Thomas [26], [27] is particularly relevant, since it gives complete answers in a number of cases. We have also included two theorems of Hirzebruch and Hopf [11] on the existence of almost complex structures and two-plane fields in compact 4-manifolds.

In Section 4 we consider what happens when $E$ cannot be embedded in the real tangent bundle. Nevertheless it can be embedded in $\mathbb{C} T \mathcal{M}$ as a sub-bundle $\mathcal{V}$ with the property that the map $\Re: \mathcal{V} \rightarrow T \mathcal{M}$ is generic. The points where $\Re: \mathcal{V} \rightarrow T \mathcal{M}$ has nontrivial kernel are the places where the multiplicity of the characteristic set of $\mathcal{V}$ changes, and are therefore of importance in PDEs.

In Section 5 we take on again the problem of embeddability of complex line bundles, this time on manifolds of dimension 2 and 3. We present other proofs of embeddability when the manifold is compact and orientable and give another, elementary proof of a result of Thomas [27].

Section 6, where the manifold is assumed to be a compact orientable surface, follows Section 4 in spirit in that we determine a number (an integer) as a measure of the characteristic set of a generically embedded bundle $\mathcal{V} \subset \mathbb{C} T \mathcal{M}$. This number is determined from the way $\mathcal{V}$ is embedded in $\mathbb{C} T \mathcal{M}$ over the characteristic points.

Sections 7 and 8 are more directly related to other aspects of the PDEs arising from involutive structures. In Section 7 we consider hypo-complex structures on an orientable 2-manifold. We recast a result of Treves [30] in a way (see Theorem 7.3) that makes it easy to construct all global hypo-complex structures on such manifolds. Finally, in Section 8 we show that, at least for rank 1 sub-bundles of $\mathbb{C} T \mathcal{M}, \mathcal{M}$ of any dimension, there is no topological obstruction to the existence of hypo-analytic structures. This is in fact just the observation that once $\mathcal{M}$ is given an analytic structure, the bundle $\mathcal{V}$ can be slightly deformed to an analytic one. We also give an alternate proof in the case of a 2-manifold. It is not known whether a given locally integrable sub-bundle always admits a hypo-analytic structure, and it is not known whether a given locally integrable sub-bundle of rank $\geq 2$ can be perturbed to obtain an analytic involutive sub-bundle.

\section{EMBEDDiNGS}

Let $\mathcal{M}$ be an $n$-manifold and let $E, F \rightarrow \mathcal{M}$ be complex vector bundles of respective ranks $r$ and $s$ with $r \leq s$. Fix Hermitian metrics on $E$ and $F$. Any injective homomorphism $E \rightarrow F$ is homotopic through injective homomorphisms 
to an isometry $\phi: E \rightarrow F$. Indeed, there is an $R: E \rightarrow E$ selfadjoint positive such that $R^{2}=\phi^{*} \phi$. Let $\phi_{1}=\phi R^{-1}$. Then $\phi_{1}$ is an isometry and

$$
\phi_{t}=\phi R^{-1}(t \mathrm{Id}+(1-t) R)
$$

is a homotopy from $\phi$ to $\phi_{1}$. So we let $\pi: \operatorname{Isom}(E, F) \rightarrow \mathcal{M}$ be the bundle whose fiber over $p \in \mathcal{M}$ is the space of isometries $E_{p} \rightarrow F_{p}$ and look for a section of this bundle. Fixing orthonormal bases of $E_{p}$ and $F_{p}$ we see that the fiber of $\operatorname{Isom}(E, F)$ is diffeomorphic to the Stiefel variety $\mathrm{V}_{r}\left(\mathbb{C}^{s}\right)$, the manifold of $r$-orthonormal frames of $\mathbb{C}^{s}$, which is $2(s-r)$-connected (see Husemoller [13, p. 92]).

Theorem 2.2. Suppose that $E$ and $F$ are complex vector bundles as above. If $r \leq s-(n-1) / 2$, then there is an isometry $\phi: E \rightarrow F$. Furthermore, if $n$ is even, or if $n$ is odd and $r \leq s-(n+1) / 2$, then any two isometries $E \rightarrow F$ are homotopic through isometries.

The proof is an application of Theorem 7.1 in Husemoller [13, p. 21]). The condition $r \leq s-(n-1) / 2$ is equivalent to $n-1 \leq 2(s-r)$, which gives the existence of the isometry, because of the connectedness of the fiber. If $n$ is even, this condition is also equivalent to $n \leq 2(s-r)$, which gives the existence of homotopies between any two embeddings. Also, if $n$ is odd, the condition $r \leq s-(n+1) / 2$ is again equivalent to $n \leq 2(s-r)$. The first part of the theorem gives the well-known result that complex vector bundles $F \rightarrow \mathcal{M}$ split as $F=F^{\prime} \oplus \mathbb{C}^{r}, \operatorname{rank} F^{\prime}=\llbracket n / 2 \rrbracket$ (see [13, Theorem 1.2, p. 99]. The second part gives stability ([13, Theorem 1.5, p. 100]).

Corollary 2.3. Let $E \rightarrow \mathcal{M}$ have rank $r \leq(n+1) / 2$. Then $E$ can be realized as a sub-bundle of $\mathbb{C} T \mathcal{M}$.

Corollary 2.4. Let $\mathcal{M}$ be even dimensional, let $E \rightarrow \mathcal{M}$ have rank $r \leq n / 2$. Then $E$ can be realized as a sub-bundle of $\mathbb{C} T \mathcal{M}$, and any two such embeddings are homotopic.

Corollary 2.5. Let $\mathcal{M}$ be odd dimensional, let $E \rightarrow \mathcal{M}$ have rank $r \leq(n+1) / 2$. Then $E$ can be realized as a sub-bundle of $\mathbb{C} T \mathcal{M}$. If $r \leq(n-1) / 2$, any two embeddings of $E$ in $\mathbb{C} T \mathcal{M}$ are homotopic.

Suppose the complex vector bundle $E \rightarrow \mathcal{M}$ of rank $r \leq(n+1) / 2$ is embedded in $\mathbb{C} T \mathcal{M}$. Let $E^{\prime}$ be complementary to $E$ in $\mathbb{C} T \mathcal{M}$. Then $2\left(\operatorname{rank} E^{\prime}\right) \geq n-1$; so automatically all elements of degree $>\operatorname{rank} E^{\prime}$ in the ring Chern $\left(E^{\prime}\right)$ (in real cohomology) generated by the Chern classes of $E^{\prime}$ are zero. Thus Bott's necessary condition for $E$ to be isomorphic to an involutive sub-bundle, namely the vanishing of the terms of degree $>n-r$ in the ring $\operatorname{Chern}\left(E^{\prime}\right)$, is trivially satisfied; so also are Shulman's conditions.

Apparently there is no example of a sub-bundle of $\mathbb{C} T \mathcal{M}$ of rank $\leq(n+1) / 2$ that is not homotopic to an involutive sub-bundle.

\section{CR STRUCTURES OF RANK 1}

If $\mathcal{V} \subset \mathbb{C} T \mathcal{M}$ is an involutive structure, then the behavior of the associated complex of differential operators is primarily controlled by the characteristic set of $\mathcal{V}$. This set, denoted by Char $\mathcal{V}$, is the union over $p \in \mathcal{M}$ of the sets

$$
\operatorname{Char}_{p} \mathcal{V}=\left\{\xi \in T_{p}^{*} \mathcal{M}:\langle\xi, v\rangle=0 \text { for all } v \in \mathcal{V}_{p}\right\} \text {. }
$$


This is a vector space for each $p$, and $\operatorname{dim} \operatorname{Char}_{p} \mathcal{V} \geq d_{\min }=\max (0, n-2 \operatorname{rank} \mathcal{V})$. So, if 2 rank $\mathcal{V}<n$, then $\operatorname{Char}_{p} \mathcal{V}$ contains nonzero covectors. In general, if $2 \operatorname{rank} \mathcal{V} \leq n$, then there may exist points $p$ where $\operatorname{dim} \operatorname{Char}_{p} \mathcal{V}>d_{\text {min }}$. These are the points $p$ such that $\mathcal{V}_{p} \cap T_{p} \mathcal{M} \neq 0$, equivalently, the points such that $\Re: \mathcal{V}_{p} \rightarrow T_{p} \mathcal{M}$ has nontrivial kernel.

A complex structure on $\mathcal{M}$ is an involutive sub-bundle $\mathcal{V} \subset \mathbb{C} T \mathcal{M}$ with $\mathcal{V} \oplus \overline{\mathcal{V}}=$ $\mathbb{C} T \mathcal{M}$. Its characteristic set is just the zero section of $T^{*} \mathcal{M}$. A generalization of this, again with trivial characteristic set, is an elliptic structure: $\mathcal{V}+\overline{\mathcal{V}}=\mathbb{C} T \mathcal{M}$ (see [15, 21]). When $\operatorname{rank}(\mathcal{V} \cap \overline{\mathcal{V}})>0$, the elliptic structure corresponds to a transversally holomorphic foliation.

A CR structure on a manifold $\mathcal{M}$ is an involutive sub-bundle $\mathcal{V} \subset \mathbb{C} T \mathcal{M}$ such that $\mathcal{V} \cap \overline{\mathcal{V}}=0$. By the usual convention, an almost CR structure is defined by omitting the requirement of involutivity. If $\mathcal{V}$ is an almost CR structure, Char $\mathcal{V}$ is a sub-bundle of $T^{*} \mathcal{M}$; its rank, $n-2 \operatorname{rank} \mathcal{V}$, is called the CR codimension of $\mathcal{V}$. An almost CR structure of rank 1 is involutive, and therefore is a CR structure.

Since the condition $\mathcal{V} \cap \overline{\mathcal{V}}=0$ implies $2 \operatorname{rank} \mathcal{V} \leq n$, Corollaries 2.4 and 2.5 give the following:

Corollary 3.1. Suppose $E \rightarrow \mathcal{M}$ can be embedded in two ways as an almost $C R$ structure. Then the two embeddings are homotopic (though not necessarily through CR structures).

Note that our definition of almost CR structures includes almost complex structures.

In Example 5.9 we present a pair of CR structures on $S^{3}$ that are not homotopic to each other through CR structures, although they are isomorphic as complex line bundles and so are homotopic as sub-bundles.

We now recall from MacPherson [17], [18] the definition of a generic homomorphism between real or complex vector bundles. Let $E, F \rightarrow \mathcal{M}$ be vector bundles. The sets

$$
\Sigma_{k}(E, F)=\left\{\psi \in \operatorname{Hom}\left(E_{p}, F_{p}\right): p \in \mathcal{M}, \operatorname{dim} \operatorname{ker} \psi=k\right\}
$$

are submanifolds of $\operatorname{Hom}(E, F)$, of codimension $k(\operatorname{rank} F-\operatorname{rank} E+k)$ if $E$ and $F$ are real, and codimension $2 k(\operatorname{rank} F-\operatorname{rank} E+k)$ if $E$ and $F$ are complex vector bundles. A smooth homomorphism $\psi: E \rightarrow F$ is said to be generic if it, as a section of $\operatorname{Hom}(E, F)$, has transversal intersection with each of the submanifolds $\Sigma_{k}(E, F)$. If the homomorphism $\psi: E \rightarrow F$ is generic, then each of the sets

$$
\Sigma_{k}(\psi)=\left\{p: \operatorname{dim} \operatorname{ker}\left(\psi_{p}: E_{p} \rightarrow F_{p}\right)=k\right\}
$$

is a submanifold of $\mathcal{M}$ whose codimension is given by the formula above.

Suppose for the rest of this section that $F_{0} \rightarrow \mathcal{M}$ is a real vector bundle of rank $s$. Let $F=\mathbb{C} F_{0}$. Suppose $E \rightarrow \mathcal{M}$ is a complex vector bundle of complex rank $r \leq s-(n-1) / 2$ as in Theorem 2.2 Let $E_{\mathbb{R}}$ be the underlying real bundle of $E$. By a generic embedding $\phi: E \rightarrow F$ we mean a smooth injective homomorphism $E \rightarrow F$ such that $\Re \phi: E_{\mathbb{R}} \rightarrow F_{0}$ is generic. Note that we distinguish generic and generic embedding. The latter is, to repeat, an embedding whose real part is generic. If $\phi$ is a generic embedding, the sets

$$
\Sigma_{k}^{\mathbb{R}}(\phi)=\Sigma_{k}(\Re \phi)
$$

are submanifolds of $\mathcal{M}$ of codimension $k(s-2 r+k), k \geq d_{\min }=\max (0,2 r-s)$. For example, if $E_{\mathbb{R}}$ is of rank 2 (i.e., $r=1$ ), the statement that $\Re \phi: E_{\mathbb{R}} \rightarrow F_{0}$ is generic 
means that for every $p \in \Sigma_{1}(\Re \phi)$, if $\sigma_{1}, \sigma_{2}$ is a frame for $E_{\mathbb{R}}$ and $\eta_{1}, \ldots, \eta_{s}$ one for $F_{0}$ (both near $p$ ) with $\Re \phi\left(\sigma_{1}\right)=\eta_{1}$ and $\Re \phi\left(\sigma_{2}\right)=\sum_{j=2}^{s} a_{j} \eta_{j}$, then the functions $a_{j}$ have independent differentials at $p$ (so are local defining functions for $\Sigma_{1}(\Re \phi)$ near $p$ ). In particular, if $s=n$, then $\Sigma_{1}^{\mathbb{R}}(\phi)$ is one-dimensional.

If $\psi: E_{\mathbb{R}} \rightarrow F_{0}$ is a bundle homomorphism, then $\phi: E \rightarrow F$ defined by

$$
\phi(\sigma)=\psi(\sigma)-\sqrt{-1} \psi(\sqrt{-1} \sigma)
$$

is $\mathbb{C}$-linear. It is an embedding if and only if for each $p$, the kernel of $\psi_{p}: E_{\mathbb{R} p} \rightarrow F_{0 p}$ is totally real.

Lemma 3.3. The set of smooth generic embeddings $\phi: E \rightarrow F$ is open and dense in the set of all smooth vector bundle maps $E \rightarrow F$ in the Whitney $C^{\infty}$ topology.

Proof. Let $\phi_{0}: E \rightarrow F$ be arbitrary. There is a generic vector bundle map $\psi_{1}$ : $E_{\mathbb{R}} \rightarrow F_{0}$ close to $\Re \phi_{0}$. Via the formula in (3.2), $\psi_{1}$ gives a complex homomorphism $\phi_{1}: E \rightarrow F$. There is a generic $\phi: E \rightarrow F$ near $\phi_{1}$. But if $\phi$ is generic, then the codimension of $\Sigma_{k}(\phi)$ is $2 k(s-r+k)$. Since $2(s-r) \geq n-1$, this codimension is $>n$ if $k>0$, and so $\Sigma_{k}(\phi)$ is empty. Thus $\phi: E \rightarrow F$ is injective. The map $\phi$ can be chosen in any prearranged neighborhood of $\phi_{0}$.

Note that if $\mathcal{M}$ is a compact real analytic manifold and $E \rightarrow \mathcal{M}$ a real or complex vector bundle, then $E$ can be given an analytic structure. If also $F \rightarrow \mathcal{M}$ is analytic and $\phi: E \rightarrow F$ a continuous homomorphism, then $\phi$ can be approximated by an analytic homomorphism. If $\phi$ is generic, or a generic embedding, then this approximation can also be taken to be generic, or a generic embedding.

We are primarily interested in the case $r=1$ and $s=n$; so suppose $2 r \leq s$. In this case we will say that $E$ embeds in $F$ without real points if there is $\phi: E \rightarrow F$ such that $\Re \phi$ is injective. If $\psi: E_{\mathbb{R}} \rightarrow F_{0}$ is injective, then so is the map $E \rightarrow F$ defined by (3.2). If $\phi$ is an embedding without real points, then $\phi_{t}$ given by (2.1) is a homotopy of embeddings without real points.

Lemma 3.4. Let $E$ and $F=\mathbb{C} F_{0}$ be complex vector bundles with Hermitian products. Let $\phi_{0}: E \rightarrow F$ and suppose that $\Re \phi_{0}$ is injective. Then $\phi_{0}$ is homotopic to an isometry $\phi_{1}: E \rightarrow F$ through maps $\phi_{t}$ such that $\Re \phi_{t}$ is injective.

A simple necessary condition for embeddability without real points is obtained from the observation that if $E_{\mathbb{R}}$ embeds in $F_{0}$, then $F_{0}$ splits as $F_{0}=E_{\mathbb{R}} \oplus \Theta$ for some vector bundle $\Theta$ of rank $s-2 r$. Thus in the formula $w(\Theta)=w\left(F_{0}\right) w(E)^{-1}$ relating the Stiefel-Whitney classes of the bundles, the terms of degree $>s-2 r$ vanish.

Suppose $E$ has complex rank 1. Then $w_{1}(E)=0$ because $E_{\mathbb{R}}$ is orientable and so $w(E)=1+w_{2}(E)$. For $u \in H^{2}\left(\mathcal{M}, \mathbb{Z}_{2}\right)$ the term of degree $q$ in $w\left(F_{0}\right)(1+u)^{-1}$ is

$$
\vartheta_{q}\left(F_{0}, u\right)=\sum_{j+2 \ell=q} w_{j}\left(F_{0}\right) u^{\ell}
$$

Thus the conditions are

$$
\vartheta_{s-1}\left(F_{0}, w_{2}(E)\right)=0
$$

and $\vartheta_{s}\left(F_{0}, w_{2}(E)\right)=0$, which is also

$$
w_{s}\left(F_{0}\right)+\left[\vartheta_{s-2}\left(F_{0}, w_{2}(E)\right)\right] w_{2}(E)=0 .
$$


The expression in brackets is $w_{s-2}(\Theta)=\mathbf{e}(\Theta) \bmod 2$. Since the Euler classes are related by $\mathbf{e}\left(F_{0}\right)=\mathbf{e}(E) \mathbf{e}(\Theta)$, one gets that if $E_{\mathbb{R}}$ embeds in $F_{0}$, then

$$
\exists v \in H^{s-2}(\mathcal{M}, \mathbb{Z}) \text { s.t. } v \quad \bmod 2=\vartheta_{s-2}\left(F_{0}, w_{2}(E)\right) \text { and } \mathbf{e}\left(F_{0}\right)=\mathbf{e}(E) v .
$$

Lemma 3.7. Let $E \rightarrow \mathcal{M}$ be a complex line bundle, $F_{0} \rightarrow \mathcal{M}$ a real bundle of rank $s \geq(n+1) / 2$. If $E$ embeds in $\mathbb{C} F_{0}$ without real vectors, then (3.5) and (3.6) hold.

If $n$ is even $\geq 4$, then $w_{n-1}(\mathcal{M})=0$ (Massey [20]). So if $E_{\mathbb{R}}$ embeds in $T \mathcal{M}$, then condition (3.5) reduces to $w_{2}(E) \vartheta_{n-3}\left(T \mathcal{M}, w_{2}(E)\right)=0$, which is satisfied if the first Chern class $c_{1}(E) \in 2 H^{2}(\mathcal{M}, \mathbb{Z})$. Note that $\vartheta_{q}\left(F_{0}, 0\right)=w_{q}\left(F_{0}\right)$, and recall that $c_{1}(E)$ maps to $w_{2}(E)$ under reduction mod 2. This and the lemma give half of the following

Theorem 3.8 (Thomas [26]). Suppose $\mathcal{M}$ is compact orientable of dimension $n \equiv 2$ $\bmod 4$. Let $E \rightarrow \mathcal{M}$ be a complex line bundle with $c_{1}(E)=2 u, u \in H^{2}(\mathcal{M}, \mathbb{Z})$. Then $E$ embeds in $\mathbb{C T M}$ as a CR structure if and only if there is $v \in H^{n-2}(\mathcal{M}, \mathbb{Z})$ such that

$$
v \quad \bmod 2=w_{m-2}(T \mathcal{M}) \quad \text { and } \quad c_{1}(E) v=\mathbf{e}(T \mathcal{M}) .
$$

For odd-dimensional manifolds, Thomas proves the following two theorems.

Theorem 3.9 (Thomas [27]). Suppose $\mathcal{M}$ is compact orientable of dimension $n \equiv 3$ $\bmod 4$. Let $E \rightarrow \mathcal{M}$ be a complex line bundle. Then $E$ embeds in $\mathbb{C} T \mathcal{M}$ as a $C R$ structure if and only if $\vartheta_{n-1}\left(T \mathcal{M}, w_{2}(E)\right)=0$.

Theorem 3.10 (Thomas 27). Suppose $n \geq 5, n \equiv 1 \bmod 4$ and $w_{2}(\mathcal{M})=0$. Then every complex line bundle $E \rightarrow \mathcal{M}$ with $c_{1}(E) \in 2 H^{2}(\mathcal{M}, \mathbb{Z})$ embeds as a $C R$ structure if and only if $w_{n-1}(\mathcal{M})=0$ and $\sum_{i=0}^{\llbracket n / 2 \rrbracket} \operatorname{dim} H_{i}\left(\mathcal{M}, \mathbb{Z}_{2}\right) \equiv 0 \bmod 2$.

Condition (3.6) is absent from Theorem 3.9 because it is implied by condition (3.5) in the case of odd-dimensional (compact oriented) manifolds, as follows. If $\vartheta_{n-1}\left(T \mathcal{M}, w_{2}(E)\right)=0$, then $E_{\mathbb{R}}$ can be embedded in $T \mathcal{M}$ over the $(n-1)$-skeleton $K^{n-1}$ of a triangulation $K$ of $\mathcal{M}$, by Proposition 2.1 in Thomas 27]. Let $\Theta \rightarrow K^{n-1}$ be complementary to an embedding of $E_{\mathbb{R}}$ in $F_{0}$ and let $v^{\prime} \in H^{n-2}\left(K^{n-1}, \mathbb{Z}\right)$ be the Euler class of $\Theta$. Since $\Theta$ has odd rank, $2 v^{\prime}=0$. Also, $v^{\prime} \bmod 2=\iota^{*} \vartheta_{n-2}\left(T \mathcal{M}, w_{2}(E)\right)$, where $\iota: K^{n-1} \rightarrow \mathcal{M}$ is the inclusion map. Since $\iota^{*}: H^{n-2}(\mathcal{M}, R) \rightarrow H^{n-2}\left(K^{n-1}, R\right)$ is an isomorphism for any ring $R$, there is a class $v \in H^{n-2}(\mathcal{M}, \mathbb{Z})$ such that $2 v=0$ and $v \bmod 2=\vartheta_{n-2}\left(T \mathcal{M}, w_{2}(E)\right)$. Since $v$ is a torsion element, $v \mathbf{e}(E)=0=\mathbf{e}(T \mathcal{M})$. So (3.6) is satisfied by $v$.

Since $\vartheta_{2}\left(T \mathcal{M}, w_{2}(E)\right)=w_{2}(T \mathcal{M})+w_{2}(E)$, Thomas' theorem above (Theorem 3.9) gives that $\mathrm{CR}$ structures on compact orientable 3 -manifolds (so $w_{2}(\mathcal{M})=0$ ) have first Chern class in $2 H^{2}(\mathcal{M}, \mathbb{Z})$, and conversely, that for any $u \in 2 H^{2}(\mathcal{M}, \mathbb{Z})$ there is a $\mathrm{CR}$ structure with $c_{1}(E)=u$. This is also noted by Thomas (op. cit.). Below we will restate this result again twice, as Corollary 4.3 and Proposition 5.5. and give two other proofs.

Also relevant are the two theorems of Hirzebruch and Hopf cited below, and for which we give their proof for the necessity of the condition.

Let $\mathcal{M}$ be compact, orientable, of dimension 4. Suppose $T \mathcal{M}$ carries an almost complex structure giving the chosen orientation. The Pontrjagin class of $\mathcal{M}$, $p_{1}(\mathcal{M})=-c_{2}(\mathbb{C} T \mathcal{M})$, is then related to the Chern classes of $T \mathcal{M}$ by

$$
p_{1}(\mathcal{M})+2 \mathbf{e}(\mathcal{M})=c_{1}(T \mathcal{M})^{2} .
$$


Let $S: H^{2}(\mathcal{M}, \mathbb{R}) \times H^{2}(\mathcal{M}, \mathbb{R}) \rightarrow \mathbb{R}$ be the bilinear form

$$
S(\alpha, \beta)=\int_{\mathcal{M}} \alpha \wedge \beta .
$$

Let $\tau$ be the signature of $S$. Then $p_{1}(\mathcal{M})[\mathcal{M}]=3 \tau$ ([11, p. 168, Formula (ii)]). So

$$
3 \tau+2 \chi(\mathcal{M})=\int_{\mathcal{M}} c_{1}(T \mathcal{M})^{2}
$$

where $\chi(\mathcal{M})$ is the Euler characteristic of $\mathcal{M}$. Let

$$
W=\left\{w \in H^{2}(\mathcal{M}, \mathbb{Z}) \mid \forall \xi \in H^{2}(\mathcal{M}, \mathbb{Z}): S(w, \xi)-S(\xi, \xi) \equiv 0 \quad \bmod 2\right\} .
$$

Let $\Omega=\{S(w, w): w \in W\}$. Hirzebruch and Hopf prove that $\int_{\mathcal{M}} c_{1}^{2}(T \mathcal{M}) \in \Omega$ as follows. The map $H^{2}\left(\mathcal{M}, \mathbb{Z}_{2}\right) \ni v \mapsto v^{2} \in H^{4}\left(\mathcal{M}, \mathbb{Z}_{2}\right)$ is a homomorphism, so given by multiplication by an element $U_{2} \in H^{2}\left(\mathcal{M}, \mathbb{Z}_{2}\right)$. But $U_{2}=w_{2}$ (due to $\mathrm{Wu}$, see 11, pp. 168 and 169]); so

$$
v^{2}=w_{2} v \quad \forall v \in H^{2}\left(\mathcal{M}, \mathbb{Z}_{2}\right) .
$$

Since $c_{1}(T \mathcal{M}) \bmod 2=w_{2}(\mathcal{M}), c_{1}(T \mathcal{M})$ has the property

$$
\eta^{2} \equiv c_{1}(T \mathcal{M}) \eta \quad \bmod 2 \quad \forall \eta \in H^{2}(\mathcal{M}, \mathbb{Z})
$$

which means that $c_{1}(T \mathcal{M}) \in W$. This gives half of

Theorem 3.11 (Hirzebruch-Hopf [11]). Let $\mathcal{M}$ be a compact, orientable 4-manifold. Then $\mathcal{M}$ admits an almost complex structure if and only if there is $w \in W$ such that

$$
p_{1}(\mathcal{M})+2 \mathbf{e}(\mathcal{M})=w^{2} .
$$

Suppose now that $T \mathcal{M}$ admits the complex line bundle $E$ as a (real) sub-bundle. Let $\Theta$ be complementary to $E$ in $T \mathcal{M}$. Since $T \mathcal{M}$ and $E$ are oriented, $\Theta$ inherits a canonical orientation. So also $\Theta$ admits a complex structure, and therefore so does $T \mathcal{M}$, with $c_{1}(T \mathcal{M})=c_{1}(E)+c_{2}(\Theta)$ and $\mathbf{e}(T \mathcal{M})=c_{1}(E) c_{1}(\Theta)$. This gives

$$
3 \tau+2 \chi(\mathcal{M})=\int_{\mathcal{M}}\left(c_{1}(E)+c_{1}(\Theta)\right)^{2}
$$

as before, and also

$$
3 \tau-2 \chi(\mathcal{M})=\int_{\mathcal{M}}\left(c_{1}(E)-c_{1}(\Theta)\right)^{2}
$$

since $\left(c_{1}(E)+c_{1}(\Theta)\right)^{2}-\left(c_{1}(E)-c_{1}(\Theta)\right)^{2}=4 \mathbf{e}(\mathcal{M})$. Since $c_{1}(E)+c_{1}(\Theta)$ represents $w_{2}(\mathcal{M}), c_{1}(E)+c_{1}(\Theta) \in W$. Also, since $c_{1}(E)-c_{1}(\Theta) \equiv c_{1}(E)+c_{1}(\Theta) \bmod 2$, $c_{1}(E)-c_{1}(\Theta) \in W$.

Theorem 3.12 (Hirzebruch-Hopf, op. cit.). Let $\mathcal{M}$ be a compact, orientable 4manifold. Then $\mathcal{M}$ admits an orientable 2-plane bundle if and only if there are $w$, $w^{\prime} \in W$ such that

$$
p_{1}(\mathcal{M})+2 \mathbf{e}(\mathcal{M})=w^{2} \quad \text { and } \quad p_{1}(\mathcal{M})-2 \mathbf{e}(\mathcal{M})=w^{\prime 2} .
$$

For other embedding results, see Heaps [10], and, more recently, work by Čadek and Vanžura, for instance [6], [7]. 


\section{Generic embeddings of Line Bundles}

Let $E \rightarrow \mathcal{M}$ be a complex line bundle and $\phi: E \rightarrow \mathbb{C} T \mathcal{M}$ a generic embedding. The obstruction to the existence of an embedding $E_{\mathbb{R}} \rightarrow T \mathcal{M}$ gives information on the places where the dimension of $\operatorname{Char}_{p} \phi(E)$ increases. To study this we follow the classical approach to obstruction theory.

Let $E, F \rightarrow \mathcal{M}$ be real vector bundles of respective ranks $r$ and $s$ over the $n$ manifold $\mathcal{M}$. Fix inner products on $E$ and $F$. As in the complex case discussed in Section 2 any embedding of $E$ in $F$ is homotopic to an isometric embedding. So we look for obstructions to the existence of a section of $\operatorname{Isom}(E, F) \rightarrow \mathcal{M}$. The fiber of this bundle is isomorphic to the Stiefel variety $\mathrm{V}_{r}\left(\mathbb{R}^{s}\right)$, which is $(s-r-1)$-connected (Husemoller [13 p. 91])). So if $K$ is a triangulation of $\mathcal{M}$, then a section exists over the $(s-r)$-skeleton $K^{s-r}$ of $K$. Recall (see Steenrod 25], for example) that since $\mathrm{V}_{r}\left(\mathbb{R}^{s}\right)$ is $(s-r)$-simple, there is a bundle of coefficients $\Pi_{s-r} \operatorname{Isom}(E, F)$ with fiber the $(s-r)$-th homotopy group of the fibers of $\operatorname{Isom}(E, F)$, and that, therefore, a section of $\operatorname{Isom}(E, F)$ over $K^{s-r}$ defines an element $\mathfrak{o}_{s-r+1}(E, F) \in$ $H^{s-r+1}\left(\mathcal{M}, \Pi_{s-r} \operatorname{Isom}(E, F)\right)$ whose vanishing is equivalent to the existence of a section over $K^{s-r+1}$.

In the case of interest here, $s=n$ and $r=2$, the fiber of $\Pi_{n-2} \operatorname{Isom}(E, F)$, $\pi_{n-2}\left(\mathrm{~V}_{2}\left(\mathbb{R}^{n}\right)\right)$, is

$$
G= \begin{cases}\mathbb{Z} & n \text { even, } n>2, \\ \mathbb{Z}_{2} & n \text { odd. }\end{cases}
$$

So if $n$ is odd, the bundle of coefficients is trivial, and $\mathfrak{o}_{n-1}(E, F)$ can be regarded as an element in $H^{n-1}\left(\mathcal{M}, \mathbb{Z}_{2}\right)$. If $n$ is even and greater than 2 , then $\Pi_{n-2} \operatorname{Isom}(E, F)$ is trivial if $E$ and $F$ are orientable. Assume then that $E$ and $F$ are orientable in this case, and fix orientations for these vector bundles. This gives a specific isomorphism $\Pi_{n-2} \operatorname{Isom}(E, F) \rightarrow \mathcal{M} \times \mathbb{Z}$, which again allows $\mathfrak{o}_{n-1}(E, F)$ to be regarded as an element of $H^{n-1}(\mathcal{M}, \mathbb{Z})$.

Thus the primary obstruction to the existence of an embedding $E \rightarrow F$ is an element of $H^{n-1}(\mathcal{M}, G)$. For embeddings of nonorientable 2-plane bundles see Mello [19, where local coefficients cannot be avoided. Now take $\mathcal{M}$ compact $n$ dimensional and, if $n$ is even, orientable. This obstruction corresponds by Poincaré duality to some element $b \in H_{1}(\mathcal{M}, G)$. We give a geometric construction of $b$ by relating it to the singular set of a generic bundle map $E \rightarrow F$.

Let then $\psi: E \rightarrow F$ be a generic map. The set $\Sigma \subset \mathcal{M}$ at which $\psi$ fails to be an embedding is a submanifold of dimension 1 (cf. Section 3). Since we are taking $\mathcal{M}$ compact, $\Sigma$ is a finite union of disjoint circles.

Let $\gamma_{j}$ be one of those circles. Choose some $p_{j} \in \gamma_{j}$ and trivializations of $E$ and $F$ near $p_{j}$. Let $U$ be a small hypersurface intersecting $\gamma_{j}$ transversally at $p_{j}$ and let $S_{0}^{n-2}$ be a small sphere in $U$ surrounding $p_{j}$. For each $p \in S_{0}^{n-2}$ we have that $\psi: E_{p} \rightarrow F_{p}$ is an embedding. Using orthonormal trivializations (oriented if $n$ is even), we replace this map by $\Psi: S_{0}^{n-2} \rightarrow \mathrm{V}_{2}\left(\mathbb{R}^{n}\right)$.

When $n$ is even we assume that $\mathcal{M}$ is oriented and that each $\gamma_{j}$ is (arbitrarily) assigned some orientation. These then induce an orientation on $U$ and hence also on $S_{0}^{n-2}$. Let $\theta: S^{n-2} \rightarrow S_{0}^{n-2}$ be any diffeomorphism from the standard sphere (of degree +1 , when $n$ is even). We see that $\Psi \circ \theta$ defines an element $b_{j}$ of $\pi_{n-2}\left(\mathrm{~V}_{2}\left(\mathbb{R}^{n}\right)\right)$ that is independent of the choice of $p_{j} \in \gamma_{j}$ and that, when $n$ is even, $b_{j}$ changes 
sign as the orientation of $\gamma_{j}$ is reversed. Let $\left[\gamma_{j}\right] \in H_{1}(\mathcal{M}, G)$ be the homology class represented by $\gamma_{j}$.

Theorem 4.1. Let $\mathcal{M}$ be compact of dimension $n \geq 3$, let $E, F \rightarrow \mathcal{M}$ be real vector bundles of respective ranks 2 and $n$. If $n$ is even, assume that $E, F$ and $\mathcal{M}$ are oriented. The primary obstruction to the existence of an embedding $E \hookrightarrow F$ is the Poincaré dual of $b=\Sigma b_{j}\left[\gamma_{j}\right] \in H_{1}(\mathcal{M}, G)$.

Proof. We begin with a generic $\psi: E \rightarrow F$ with singular set $\Sigma=\bigcup \gamma_{j}$. Let $K$ be a triangulation of $\mathcal{M}$. Perturb $\psi$, if necessary, to achieve that $\Sigma$ avoids $K^{n-2}$ and is transverse to any $(n-1)$-simplex it intersects. Recall the construction of the obstruction class: Let $\sigma$ be any oriented $(n-1)$-simplex in $K$. We have a map $f: \partial \sigma \rightarrow \mathrm{V}_{2}\left(\mathbb{R}^{n}\right)$ which comes from the embedding $E \hookrightarrow F$ on $K^{n-2}$ and a choice of trivializations over $\sigma$ of $E$ and $F$ (respecting orientations if $n$ is even). As above, $f$ determines an element of $\pi_{n-2}\left(\mathrm{~V}_{2}\left(\mathbb{R}^{n}\right)\right.$ ) (note that we are implicitly using that $\partial \sigma$ inherits an orientation from that of $\sigma$ ). Thus we assign an element of $G$ to any $(n-1)$-simplex. That is, we have defined an $(n-1)$-cochain $c^{n-1}$ with values in $G$. The usual argument shows that this cochain is closed and so defines a cohomology class $\left[c^{n-1}\right]=\mathfrak{o}_{n-1}(E, F) \in H^{n-1}(\mathcal{M}, G)$. This is the primary obstruction class for embedding $E$ into $F$.

We now relate $\mathfrak{o}_{n-1}(E, F) \in H^{n-1}(\mathcal{M}, G)$ to $b \in H_{1}(\mathcal{M}, G)$. If $\sigma$ does not intersect $\gamma_{j}$, then the embedding $E \hookrightarrow F$ over $K^{n-2}$ extends over $\sigma$ and so $c^{n-1}(\sigma)=0$; if $\sigma$ does intersect $\gamma_{j}$, then the intersection is transverse and $c^{n-1}(\sigma)= \pm b_{j}$. When $n$ is odd, the ambiguity in sign is irrelevant. When $n$ is even, the sign is positive if the orientation of $\gamma_{j}$ induces on $\partial \sigma$ the same orientation as the one inherited from the orientation of $\sigma$. Also, in this case, the intersection number of $\sigma^{n-1}$ and $\gamma_{j}$ is +1 . So, in any event, $c^{n-1}(\sigma)=b_{j} \sigma^{n-1} \cdot \gamma_{j}$. Thus for any closed $(n-1)$-chain $\mathrm{s}$ we have

$$
c^{n-1}(\mathrm{~s})=\mathrm{s} \cdot \Sigma b_{j} \gamma_{j} .
$$

Passing to cohomology we see that indeed $\left[c^{n-1}\right]$ is the Poincaré dual of $b$.

The results for $n=2$ are somewhat different. First, the group $G$ is now $\pi_{0}\left(\mathrm{~V}_{2}\left(\mathbb{R}^{2}\right)\right.$ ), which we take to be $\mathbb{Z}_{2}$. (The two components of $\mathrm{V}_{2}\left(\mathbb{R}^{2}\right)$ correspond to the two possible orientations of the 2 -frame. We arbitrarily choose one component to represent $0 \in \mathbb{Z}_{2}$.) This contrasts with the fact that $G=\mathbb{Z}$ for $n$ even and greater than 2. As before, we start with an embedding $E \hookrightarrow F$ on the $(n-2)$-skeleton. For $n=2$, this means we give embeddings at each vertex of the triangulation. Let us first assume that $E$ and $F$ are oriented. Then we change whatever is necessary to make all the maps $E \hookrightarrow F$ at the vertices orientation-preserving. Then we may extend these maps to $E \hookrightarrow F$ over $K^{1}$. Hence the primary obstruction $\mathfrak{o}_{1}$ is zero. Also, starting with a map $E \hookrightarrow F$ on a neighborhood of $K^{1}$, we may find a generic map with each $\gamma_{j}$ contained in a 2-simplex of the triangulation. So $\left[\gamma_{j}\right]=0$ and thus $b=0$. So Theorem 4.1 holds because both quantities are zero. Next, consider $E, F$ and $\mathcal{M}$ not oriented. Now $\mathfrak{o}_{1}$ need not be zero and the $\gamma_{j}$ need not lie in 2simplices. The previous proof for the non-oriented (odd) case remains valid, and so $\mathfrak{o}_{1}=b \in H^{1}\left(\mathcal{M}^{2}, \mathbb{Z}_{2}\right)$. In conclusion, the two-dimensional case has features of the general even- and odd-dimensional cases.

Next we look at the primary and secondary obstructions in dimensions two and three for orientable bundles. We have seen that there is no primary obstruction when $n=2$, and we shall show that there is no secondary obstruction when $n=3$. 
While the primary obstruction to extending $E^{2} \hookrightarrow F^{2}$ from $K^{0}$ to $K^{1}$ for orientable bundles vanishes, there clearly is a secondary obstruction (given by the difference of the Chern classes) for extending the embedding to all of $\mathcal{M}$. This obstruction can be computed directly from the winding of the range of $\psi$ around the singularity curves. We omit the argument, only saying that it reduces to a simple calculation for some standard examples. A more interesting result starts with a generic $E \rightarrow F$ and its characteristic set $\Sigma$, rather than building up along the skeleton. Then the components of $\Sigma$ need not lie in coordinate patches. This is done for $F=T \mathcal{M}$ in Theorem 6.6 below.

In dimension 3 , the primary obstruction can be computed directly and, furthermore, it is the only obstruction.

Theorem 4.2. Let $E$ and $F$ be orientable real vector bundles over an orientable compact 3-manifold $\mathcal{M}$, rank $E=2$, $\operatorname{rank} F=3$. The only obstruction to an embedding $E \hookrightarrow F$ is given by $w_{2}(F)-w_{2}(E)$.

Corollary 4.3. $E^{2}$ embeds into $T \mathcal{M}^{3}$ if and only if $c_{1}(E) \in 2 H^{2}(\mathcal{M}, \mathbb{Z})$.

Remark 4.4. We have already pointed out that this result follows from Theorem 3.9 .

Proof. Let $\mathfrak{o}_{2} \in H^{2}\left(\mathcal{M}, \mathbb{Z}_{2}\right)$ be the primary obstruction to finding an embedding $E \hookrightarrow F$ on $K^{2}$. Let $\lambda: H^{2}(\mathcal{M}, \mathbb{Z}) \rightarrow H^{2}\left(\mathcal{M}, \mathbb{Z}_{2}\right)$ be the map induced by $\mathbb{Z} \rightarrow \mathbb{Z}_{2}$ and let $\tilde{E}$ be any complex line bundle on $\mathcal{M}$ with $\lambda\left(c_{1}(\tilde{E})-c_{1}(E)\right)=\mathfrak{o}_{2}$. On $K^{1}$ we have embeddings $\tilde{E} \hookrightarrow E$ and $E \hookrightarrow \mathrm{V}_{2}\left(\mathbb{R}^{3}\right)$ and the composition $\tilde{E} \hookrightarrow$ $\mathrm{V}_{2}\left(\mathbb{R}^{3}\right)$. Denote the obstructions to extending these maps to $K^{2}$ by $k \in H^{2}(\mathcal{M}, \mathbb{Z})$, $\mathfrak{o}_{2} \in H^{2}\left(\mathcal{M}, \pi_{1}\left(\mathrm{~V}_{2}\left(\mathbb{R}^{3}\right)\right)\right)$, and $\tilde{c} \in H^{2}\left(\mathcal{M}, \pi_{1}\left(\mathrm{~V}_{2}\left(\mathbb{R}^{3}\right)\right)\right)$. Fix some 2-simplex $\sigma^{2}$ and trivializations for $\tilde{E}, E, F$. These give rise to maps

$$
f: S^{1} \rightarrow S O(2), \quad g: S^{1} \rightarrow \mathrm{V}_{2}\left(\mathbb{R}^{3}\right)=S O(3), \quad h: S^{1} \rightarrow S O(3) .
$$

Let $\mu: S O(2) \rightarrow S O(3)$ be the map $S O(2) \rightarrow S O(2) \oplus 1 \subset S O(3)$. Then for each $\theta \in S^{1}, h(\theta)=\mu(f(\theta)) \cdot g(\theta)$ where we are using group multiplication. Denote by $[h]$ the homotopy class of $h \in \pi_{1}\left(\mathrm{~V}_{2}\left(\mathbb{R}^{3}\right)\right)$. By a basic result in the homotopy of topological groups, $[h]=[\mu f]+[g]$. Doing this for each $\sigma^{2}$ gives cocycles and, passing to cohomology, we have

$$
\tilde{c}=c_{\mu f}+c .
$$

Note that $c_{\mu f} \in H^{2}\left(\mathcal{M}, \pi_{1}\left(\mathrm{~V}_{2}\left(\mathbb{R}^{3}\right)\right)\right) \cong H^{2}\left(\mathcal{M}, \mathbb{Z}_{2}\right)$ is the image under the map $\lambda: H^{2}(\mathcal{M}, \mathbb{Z}) \rightarrow H^{2}\left(\mathcal{M}, \mathbb{Z}_{2}\right)$ of the obstruction $c_{f} \in H^{2}(\mathcal{M}, \mathbb{Z})$ to extending $f$ to $K^{2}$. Furthermore, this obstruction is up to sign the difference of the Chern classes $c_{1}(\tilde{E})-c_{1}(E)$. Thus

$$
\begin{aligned}
\tilde{c} & =\lambda\left(c_{1}(\tilde{E})\right)-c_{1}(E)+c \\
& =c+c \\
& =0 .
\end{aligned}
$$

Hence, there is no obstruction to embedding $\tilde{E} \hookrightarrow F$ on $K^{2}$. Since $\pi_{2}\left(\mathrm{~V}_{2}\left(\mathbb{R}^{3}\right)\right)=0$, there is no further obstruction and one can embed $\tilde{E} \hookrightarrow F$ on $\mathcal{M}$. Thinking of $\tilde{E} \subset$ $F$, we may write $F=\tilde{E} \oplus \mathbb{R}$. Thus, $w_{2}(\tilde{E})=w_{2}(F)$ and so $\mathfrak{o}_{2}=w_{2}(F)-w_{2}(E)$. 


\section{Line Sub-Bundles of $\mathbb{C} T \mathcal{M}$ In LOW-DIMENSION}

Complex vector bundles over 2- or 3-manifolds are classified by their rank and first Chern class, since line bundles are classified by their Chern class and any complex vector bundle $F \rightarrow \mathcal{M}$ of rank $r$ splits as $F=L \oplus \mathbb{C}^{r-1}$ over such manifolds. This permits an alternate approach to proving embeddability of complex line subbundles in $\mathbb{C} T \mathcal{M}$ in these low-dimensional manifolds.

For reference we state

Lemma 5.1. Let $\mathcal{M}$ be 2- or 3-dimensional. Let $E_{1}, E_{2} \rightarrow \mathcal{M}$ be complex vector bundles of the same rank. If $c_{1}\left(E_{1}\right)=c_{1}\left(E_{2}\right)$, then $E_{1}=E_{2}$.

Proposition 5.2. $\mathbb{C} T \mathcal{M}$ is a trivial vector bundle for any compact orientable twoor three-dimensional manifold $\mathcal{M}$.

Indeed, if $\mathcal{M}$ is an orientable 3 -manifold, then $T \mathcal{M}$ itself is trivial. In general, if $F_{0} \rightarrow \mathcal{M}$ is a real vector bundle, then $2 c_{2 j+1}\left(\mathbb{C} F_{0}\right)=0$. If $\mathcal{M}$ is compact orientable of dimension 2 , this gives $c_{1}\left(\mathbb{C} F_{0}\right)=0$ since $H^{2}(\mathcal{M}, \mathbb{Z})=\mathbb{Z}$.

More generally, a result of Peterson [23] (see also Kervaire [16]) shows that $\mathbb{C} T \mathcal{M}$ is trivial whenever $c(\mathbb{C} T \mathcal{M})=1$ except possibly when there exists some nonzero $\eta \in H^{2 j}(\mathcal{M}, \mathbb{Z}), \eta \neq 0$, with $(j-1) ! \eta=0$. So, for instance, if $\mathcal{M}^{n}$ is orientable and immerses in $\mathbb{R}^{n+1}$, then $\mathbb{C} T \mathcal{M}$ is trivial, except possibly when there exists such a torsion element. Of course, triviality fails when the total Chern class is not 1 , for example, for $\mathbb{C} T\left(\mathbb{C P}^{n}\right)$ whenever $n>1$.

Using Proposition [5.2 we can reprove Corollary 2.3 for line bundles over orientable manifolds as follows. Let $E \rightarrow \mathcal{M}$ be complex line bundle. Then $E \oplus \bar{E}$ has first Chern class equal to 0 , so is trivial. So if $\mathcal{M}$ is 2 -dimensional, then $E \oplus \bar{E}$ is isomorphic to $\mathbb{C} T \mathcal{M}$ and therefore $E$ embeds in $\mathbb{C} T \mathcal{M}$. The same argument works in the 3-dimensional case. In fact, even if $\mathcal{M}^{3}$ is not orientable, $\mathbb{C} T \mathcal{M} \cong L \otimes \mathbb{C}^{2}$, and so again $E \oplus \bar{E}$ can be embedded in $\mathbb{C} T \mathcal{M}$.

Proposition 5.3. Any complex line bundle E over a 2-or 3-dimensional manifold $\mathcal{M}$ is isomorphic to a sub-bundle of $\mathbb{C} T \mathcal{M}$.

One can also get the embeddings of rank 2 complex bundles in $\mathbb{C} T \mathcal{M}$ in the case of 3-manifolds, as follows. Suppose $\mathcal{M}$ is 3-dimensional, orientable or not, let $E \rightarrow \mathcal{M}$ have rank 2 . Let $\mu=c_{1}(\mathbb{C} T \mathcal{M})-c_{1}(E)$. Let $L \rightarrow \mathcal{M}$ be the line bundle with $c_{1}(L)=\mu$. Then $L$ can be realized as a sub-bundle of $\mathbb{C} T \mathcal{M}$, and admits a complementary bundle $E^{\prime}$. Consequently, $c\left(E^{\prime}\right) c(L)$ and $c(E) c(L)$ are equal to $c(\mathbb{C} T \mathcal{M})$; so $c(E)=c\left(E^{\prime}\right)$. Thus $E$ is isomorphic to $E^{\prime} \subset \mathbb{C} T \mathcal{M}$ by Lemma 5.1

Proposition 5.4. If $\operatorname{dim} \mathcal{M}=3$, any complex rank 2 vector bundle $E \rightarrow \mathcal{M}$ is isomorphic to a sub-bundle $\mathcal{W}$ of $\mathbb{C} T \mathcal{M}$.

We now give another proof of Theorem 3.9 in the case of a compact orientable 3-manifold $\mathcal{M}$. Recall that in this case the necessary and sufficient condition for a line bundle $E$ to embed as a $\mathrm{CR}$ structure is that $c_{1}(E) \in 2 H^{2}(\mathcal{M}, \mathbb{Z})$. Let $U$ be a nowhere zero vector field on $\mathcal{M}$ and let $g$ be some Riemannian metric on $\mathcal{M}$. Let $H$ denote the two-plane distribution orthogonal to $U$. Fix an orientation for $\mathcal{M}$ and let $J: H \rightarrow H$ be rotation by $\pi / 2$ in the direction chosen such that $\{U, X, J X\}$ is positively oriented for some (and therefore for all) nonzero $X \in H$. Then the pair $\{H, J\}$ defines a complex line bundle on $\mathcal{M}$ and also a CR structure with elements $X-i J X$. Denote this line bundle by $U^{\perp}$. We ask if a given line bundle $E$ can be realized in this way. 
Proposition 5.5. Let $\mathcal{M}$ be a compact, orientable, three-dimensional manifold. A necessary and sufficient condition for a complex line bundle $E$ over $\mathcal{M}$ to be isomorphic to some $U^{\perp}$ is that $c_{1}(E) \in 2 H^{2}(\mathcal{M}, \mathbb{Z})$.

Proof. Assume $E$ is isomorphic to some $U^{\perp}$. Let $\{U\}$ denote the (trivial) line bundle spanned by $U$. Then $E \oplus\{U\} \cong \mathcal{M} \times \mathbb{R}^{3}$. So, using the underlying real structure of $E$, we have for the total Stiefel-Whitney class,

$$
1=w(E \oplus\{U\})=w(E) w(\{U\}) .
$$

In particular, this gives $w_{2}(E)=0$, since $w(\{U\})=1$. Thus $c_{1}(E) \in 2 H^{2}(\mathcal{M}, \mathbb{Z})$.

For the converse, we start with a few observations. Consider $T S^{2}$ as a complex line bundle. Let $\omega$ be the positive generator of $H^{2}\left(S^{2}, \mathbb{Z}\right)$. Then

a) $c_{1}\left(T S^{2}\right)=2 \omega$

b) $\omega$ is the negative of the first Chern class of the canonical line bundle $\gamma \rightarrow$ $\mathbb{C P}^{1}$

c) complex line bundles over $\mathcal{M}^{3}$ are induced by maps into $\mathbb{C P}^{1}$.

The first observation is a special case of a general result that the top Chern class of the complex tangent bundle (as opposed to the complexified tangent bundle) of a complex manifold is the Euler class of the manifold [22, p. 158] and the second observation is one of the axioms defining Chern classes [13, p. 249].

For the third observation, consider a generic section of a line bundle $L$ on $\mathcal{M}^{3}$. This section is zero over a one-dimensional submanifold $\Sigma$ of $\mathcal{M}$. Since any complex line bundle is trivial over a small tubular neighborhood of $\Sigma$, we may find two open sets $U_{1}, U_{2} \subset \mathcal{M}$ with

$$
\mathcal{M}=U_{1} \cup U_{2} \text { and }\left.L\right|_{U_{j}} \text { trivial. }
$$

In this situation (where $\mathcal{M}$ may be of any dimension) there exists a map

$$
\Phi: \mathcal{M} \rightarrow \mathbb{C P}^{1}
$$

such that $\Phi^{*} \gamma=L$. It follows that

$$
c_{1}(L)=c_{1}\left(\Phi^{*}(\gamma)\right) .
$$

Given the bundle $E$ with $c_{1}(E)=2 \eta$ we construct $\Phi: \mathcal{M}^{3} \rightarrow \mathbb{C P}^{1}$ with $\Phi^{*}(\omega)=$ $\eta$ (by applying the third observation to the line bundle $L$ whose Chern class is $-\eta$ ). We claim that $E$ is isomorphic to $\Phi^{*}\left(T S^{2}\right)$ and that $\Phi^{*}\left(T S^{2}\right)$ is isomorphic to a sub-bundle of $\mathbb{C} T \mathcal{M}$ of the type $U^{\perp}$. We have

$$
c_{1}\left(\Phi^{*}\left(T S^{2}\right)\right)=\Phi^{*}\left(c_{1}\left(T S^{2}\right)\right)=2 \eta=c_{1}(E) .
$$

So $E=\Phi^{*}\left(T S^{2}\right)$. If we think of $S^{2} \subset \mathbb{R}^{3}$ we may write $\Phi=\left(\phi_{1}, \phi_{2}, \phi_{3}\right)$. The bundle $\Phi^{*}\left(T S^{2}\right)$ is given by

$$
\left\{\left(p,\left.\sum_{j=1}^{3} a_{j} \partial_{\phi_{j}}\right|_{\phi(p)}\right) \in \mathcal{M} \times T S^{2}: a_{j} \in \mathbb{R}, \sum_{j} a_{j} \phi_{j}(p)=0\right\} .
$$

Let $X_{1}, X_{2}, X_{3}$ be global independent vector fields on $\mathcal{M}$ and define $\Psi: \Phi^{*} T S^{2} \rightarrow$ $T \mathcal{M}$ by

$$
\Psi\left(p,\left.\sum_{j=1}^{3} a_{j} \partial_{\phi_{j}}\right|_{\phi(p)}\right)=\sum_{j=1}^{3} a_{j} X_{j}(p)
$$


This is injective, hence an embedding of $\Phi^{*}\left(T S^{2}\right)$ in $T \mathcal{M}$. Note that $\Phi^{*}\left(T S^{2}\right)$ is orthogonal to $U=\sum_{i} \phi_{i} X_{i}$; so, as a complex bundle, $\Phi^{*}\left(T S^{2}\right)$ is isomorphic to $U^{\perp}$ or $(-U)^{\perp}$.

We have also proved:

Lemma 5.6. Let $\mathcal{M}^{3}$ be orientable and let $\left\{X_{1}, X_{2}, X_{3}\right\}$ be an oriented global basis for TM. Define a Riemannian metric on $\mathcal{M}$ by taking this as an orthonormal basis. Let $\Phi: \mathcal{M} \rightarrow S^{2} \subset R^{3}$ have components $\Phi=\left(\phi_{1}, \phi_{2}, \phi_{3}\right)$. Set $U=\sum \phi_{i} X_{i}$. Then $U^{\perp}=\Phi^{*}\left(T S^{2}\right)$ as complex line bundles.

Remark 5.7. The sufficiency part of Theorem 5.5 is also implicit in the work of Turaev on $\operatorname{Spin}^{c}$ structures [31.

Corollary 5.8. Let $\mathcal{M}$ be a compact, orientable, three-dimensional manifold. Let $E$ be a rank 2 complex vector bundle over $\mathcal{M}$. Then $E$ is isomorphic to a sub-bundle $\mathcal{V} \subset \mathbb{C} T \mathcal{M}$ such that $\mathcal{V}+\overline{\mathcal{V}}=\mathbb{C} T \mathcal{M}$ if and only if $c_{1}(E) \in 2 H^{2}(\mathcal{M}, \mathbb{Z})$.

Proof. Fix a Riemannian metric on $\mathcal{M}$ and extend it to a Hermitian metric on $\mathbb{C} T \mathcal{M}$. Suppose that $E$ has been realized as a sub-bundle $\mathcal{V}$ such that $\mathcal{V}+\overline{\mathcal{V}}=$ $\mathbb{C} T \mathcal{M}$. Then $\mathcal{V} \cap \overline{\mathcal{V}}$ is the complexification of a real line sub-bundle $R \subset T \mathcal{M}$. Let $\mathcal{W}$ be the orthogonal of $\mathbb{C} R$ in $\mathcal{V}$. Then $\mathcal{W}$ and $\overline{\mathcal{W}}$ are CR structures. In particular, $c_{1}(\overline{\mathcal{W}}) \in 2 H^{2}(\mathcal{M}, \mathbb{Z})$. Since $0=c_{1}(\mathbb{C} T \mathcal{M})=c_{1}(\mathbb{C} R \oplus \mathcal{W})+c_{1}(\overline{\mathcal{W}})$ and $\mathbb{C} R \oplus \mathcal{W}=\mathcal{V}, c_{1}(E) \in 2 H^{2}(\mathcal{M}, \mathbb{Z})$.

Suppose now that $c_{1}(E) \in 2 H^{2}(\mathcal{M}, \mathbb{Z})$. As in the proof of Proposition 5.4 let $\mu=-c_{1}(E)$ and let $L \rightarrow \mathcal{M}$ be the line bundle with $c_{1}(L)=\mu$. Fix a Hermitian product on $L$. Since $\mu \in 2 H^{2}(\mathcal{M}, \mathbb{Z}), L$ can be realized as a CR structure on $\mathcal{M}$; so we can find an isometry $\psi: L_{\mathbb{R}} \rightarrow T \mathcal{M}$. Then $\phi: L \rightarrow \mathbb{C} T \mathcal{M}$ given by $\phi(\sigma)=\psi(\sigma)-\sqrt{-1} \psi(\sqrt{-1} \sigma)$ is an embedding. For $\sigma \in L_{p}$, we have $\phi(\sigma)=u_{1}-i u_{2}$ with $u_{j} \in T \mathcal{M}$ and $u_{1}$ and $u_{2}$ independent. Complete this to a basis $\left\{u_{1}, u_{2}, u_{3}\right\}$ of $T \mathcal{M}_{p}$. Now let $\mathcal{V}$ be the orthogonal of $\phi(L)$ in $\mathbb{C} T \mathcal{M}$. Note that $c_{1}(\mathcal{V})=c_{1}(E)$ and so $\mathcal{V}$ is isomorphic to $E$. Furthermore, $\mathcal{V}_{p}$ is spanned by $u_{1}+i u_{2}$ and $u_{3}$. So $\mathcal{V}_{p}+\overline{\mathcal{V}}_{p}=\mathbb{C} T \mathcal{M}$

We now show that a bundle might have two realizations $U^{\perp}$ and $\tilde{U}^{\perp}$ but these realizations might not be deformable into each other within this class. In particular, we find two CR structures on $S^{3}$ (each of which is trivial as a line bundle because $H^{2}\left(S^{3}, \mathbb{Z}\right)=0$ ) that are not deformable into each other through CR structures. For suppose $\Phi, \tilde{\Phi}: \mathcal{M} \rightarrow S^{2}$ are smooth (or just continuous) with components $\phi_{i}, \tilde{\phi}_{i}$. Let $U=\sum_{i} \phi_{i} X_{i}$, likewise $\tilde{U}$. If $\Phi^{*}=\tilde{\Phi}^{*}$ as maps $H^{2}\left(S^{2}, \mathbb{Z}\right) \rightarrow H^{2}(\mathcal{M}, \mathbb{Z})$, then $U^{\perp}$ is isomorphic to $\tilde{U}^{\perp}$, but unless $\Phi$ is homotopic to $\tilde{\Phi}, U^{\perp}$ cannot be deformed into $\tilde{U}^{\perp}$ within $T \mathcal{M}$. This translates to: The CR structures $\{H, J\}$ and $\{\tilde{H}, \tilde{J}\}$ are isomorphic as line bundles but may not be deformable to each other along CR structures.

Example 5.9. The trivial bundle $S^{3} \times \mathbb{C}$ can be realized in two ways as a subbundle of $T S^{3}$. One way is as the standard CR structure $\mathcal{V}$ of $S^{3} \subset \mathbb{C}^{2}$. Let $z_{1}=x_{1}+\sqrt{-1} x_{3}, z_{2}=x_{2}+\sqrt{-1} x_{4}$ be the coordinates in $\mathbb{C}^{2}$. The vector fields

$$
\begin{gathered}
X_{1}=x_{3} \partial_{x_{1}}+x_{4} \partial_{x_{2}}-x_{1} \partial_{x_{3}}-x_{2} \partial_{x_{4}}, \\
X_{2}=x_{4} \partial_{x_{1}}-x_{3} \partial_{x_{2}}+x_{2} \partial_{x_{3}}-x_{1} \partial_{x_{4}}, \\
X_{3}=x_{2} \partial_{x_{1}}-x_{1} \partial_{x_{2}}-x_{4} \partial_{x_{3}}+x_{3} \partial_{x_{4}}
\end{gathered}
$$


form a positively oriented orthonormal frame on $S^{3}$. As a sub-bundle of $\mathbb{C} T S^{3}, \mathcal{V}$ is spanned by $X_{2}+\sqrt{-1} X_{3}$. So $\mathcal{V}$ corresponds to the sub-bundle of $T S^{3}$ determined by $U^{\prime}=X_{1}$, that is, $\Phi=(1,0,0)$. Let now $\tilde{\Phi}: S^{3} \rightarrow S^{2}$ be the Hopf map. Then $\tilde{\Phi}$ is not homotopic to a constant map. So it defines a CR structure $\tilde{\mathcal{V}}$ on $S^{3}$ which cannot be deformed to the standard structure within CR structures. These two CR structures are isomorphic because $S^{3}$ admits only the trivial line bundle as a complex line bundle.

Note however that $\tilde{\mathcal{V}}$ can be connected to the standard CR structure within the larger space of deformations of complex line bundles in $\mathbb{C} T S^{3}$. This is true in a general setting, by Corollary [3.1, but also follows in this case from the fact that $\pi_{3}\left(\mathbb{C P}^{2}\right)$ is trivial.

\section{Characteristic Sets in Dimension 2}

Let $\mathcal{V}$ be a complex line bundle in $\mathbb{C} T \mathcal{M}^{2}$. The characteristic set of $\mathcal{V}$ projects onto the set of points $p$ such that $\mathcal{V}_{p} \cap T_{p} \mathcal{M} \neq\{0\}$.

Definition 6.1. $\mathcal{V}$ is real at a point $p$ and $p$ is a characteristic point of $\mathcal{V}$ if there is some vector $v \in T_{p} \mathcal{M}$ such that

$$
\mathcal{V}_{p}=\{\lambda v: \lambda \in \mathbb{C}\}
$$

We denote the set of characteristic points by $\Sigma(\mathcal{V})$.

The following three conditions are each equivalent to $\mathcal{V}$ being real at $p$.

(1) The real vector space $\left\{\Re X: X \in \mathcal{V}_{p}\right\}$ is one-dimensional.

(2) For each $X \in \mathcal{V}_{p}, \Re X$ and $\Im X$ are linearly dependent.

(3) $\mathcal{V}_{p}=\overline{\mathcal{V}}_{p}$

The set of complex lines in $\mathbb{C} T_{p} \mathcal{M}$ that are real is clearly bijective with the set of real lines in $T_{p} \mathcal{M}$ and so can be identified with a circle. We let $\mathcal{P}(\mathbb{C} T \mathcal{M}) \rightarrow \mathcal{M}$ be the bundle of all complex lines in $\mathbb{C} T \mathcal{M}$ and $B_{\mathbb{R}}=\mathcal{P}(T \mathcal{M}) \rightarrow \mathcal{M}$ be the bundle of real lines in $T \mathcal{M}$ regarded as a subset of $\mathbb{C} T \mathcal{M}$. The line bundle $\mathcal{V}$ defines a section $[\mathcal{V}]$ of $\mathcal{P}(\mathbb{C} T \mathcal{M})$, and

$$
\left[\mathcal{V}_{p}\right] \in B_{\mathbb{R}} \Longleftrightarrow \mathcal{V} \text { is real at } p .
$$

Remark 6.2. Note that $B_{\mathbb{R}}$ is a nontrivial circle bundle for all compact surfaces $\mathcal{M}$, except the torus and the Klein bottle. This is because a section of $B_{\mathbb{R}}$ would be a field of lines on $\mathcal{M}$. It is well known that only the torus admits a nowhere zero real vector field. It is equally true, although perhaps not as well known, that only the torus and the Klein bottle admit real line fields. A simple proof is to fix a metric and note that the set of unit vectors in the line field provides a double cover (connected or not) that admits a global nonzero vector field. So $\mathcal{M}$ has the torus for a connected cover and so can be only the torus or the Klein bottle. That $B_{\mathbb{R}}$ is nontrivial for compact, orientable surfaces, except on the torus, also follows from the fact that $c_{1}(B)=2 \chi(\mathcal{M})$ where $B$ is the complex line bundle determined by $B_{\mathbb{R}}$. This formula is easy to prove using that the map from the sphere bundle of $T \mathcal{M}$ to $B_{\mathbb{R}}$ is a 2 -fold covering.

The section $[\mathcal{V}]$ of $\mathcal{P}(\mathbb{C} T \mathcal{M})$ determined by a complex line bundle $\mathcal{V}$ in $\mathcal{P}(\mathbb{C} T \mathcal{M})$ may or may not intersect the circle bundle $B_{\mathbb{R}}$. For example, when $\mathcal{M}$ is oriented, the complex line bundles $T^{1,0}$ and $T^{0,1}$ corresponding respectively to a complex structure on $\mathcal{M}$, compatible with the given orientation, and a complex structure 
of the opposite orientation, give sections that do not intersect $B_{\mathbb{R}}$. Conversely, if a complex line sub-bundle does not intersect $B_{\mathbb{R}}$ it gives a complex structure of one of these two types (see Lemma 7.4).

Lemma 6.3. $B_{\mathbb{R}}$ is a smooth hypersurface in $\mathcal{P}(\mathbb{C} T \mathcal{M})$ and, when $\mathcal{M}$ is orientable, separates $\mathcal{P}(\mathbb{C} T \mathcal{M})$ into two disjoint connected components:

$$
\mathcal{P}(\mathbb{C} T \mathcal{M})=B_{+} \cup B_{\mathbb{R}} \cup B_{-} .
$$

Proof. The smoothness is easily seen in local coordinates. The decomposition follows from the immediately preceding remarks about complex structures.

From the point of view of partial differential equations, the interesting properties of $\mathcal{V}$ are determined by its behavior on the characteristic set $\Sigma=\Sigma(\mathcal{V})$. This suggests the possibility of computing the Chern class of $\mathcal{V}$ using only $\left.\mathcal{V}\right|_{\Sigma}$. This indeed can be done in the generic case on an oriented $\mathcal{M}^{2}$.

So let $\mathcal{V}$ belong to the open and dense set of complex line bundles with the property that the intersection of $[\mathcal{V}] \subset \mathcal{P}(\mathbb{C} T \mathcal{M})$ and $B_{\mathbb{R}}$ is transverse (that is, $\mathcal{V}$ is generically embedded in $\mathbb{C} T \mathcal{M}$, cf. Section 3). Thus $\Sigma$ decomposes into a union of simple closed curves

$$
\Sigma=\bigcup \gamma_{j}
$$

We assume $\mathcal{M}$ is oriented and fix a metric. This determines the sub-bundle $T^{1,0}$ of $\mathbb{C} T \mathcal{M}$. We have $T^{1,0} \perp T^{0,1}$ and if $Z \in T^{1,0}$, then $|\Re Z|=|\Im Z|$. Let $B_{+}$be the component of $\mathcal{P}(\mathbb{C} T \mathcal{M}) \backslash B_{\mathbb{R}}$ that contains $\left[T^{1,0}\right]$. Let $\mathcal{R}$ be the set of points where $[\mathcal{V}]$ belongs to $B_{+}$. In particular, $[\mathcal{V}]_{p}=\left[T^{1,0}\right]_{p}$ can only occur when $p \in \mathcal{R}$. Note also that $\mathcal{R}$ is an oriented open submanifold of $\mathcal{M}$ with boundary $\Sigma$. Since all complex line bundles on $\mathcal{M}$ become trivial when restricted to any proper open subset, we can find global smooth nonzero sections

$$
\tau: \overline{\mathcal{R}} \rightarrow T^{1,0} \quad \text { and } \quad \zeta: \overline{\mathcal{R}} \rightarrow \mathcal{V} .
$$

Set

$$
\beta(p)=\frac{1}{|\zeta(p)|^{2}}\langle\tau(p), \zeta(p)\rangle\langle\tau(p), \overline{\zeta(p)}\rangle
$$

and

$$
k_{j}=\frac{1}{2 \pi i} \int_{\gamma_{j}} \frac{d \beta}{\beta} \text { and } k=\sum k_{j},
$$

where $\Sigma$ has the orientation induced by $\mathcal{R}$. It is immediate that each $k_{j}$ is independent of the choices of nonzero sections. On $\Sigma, \mathcal{V}=\{\lambda X: \lambda \in \mathbb{C}\}$ where $X$ is a real vector and $k$ may be computed using $X$ in place of $\zeta$. Thus we need to "know" only the section $\tau$.

Theorem 6.6. $\operatorname{deg}(\mathcal{V})=-k-\chi(\mathcal{M})$.

Here $\chi(\mathcal{M})$, as before, is the Euler characteristic of $\mathcal{M}$. Recall the construction of a line bundle on $S^{2}$ by means of a gluing function. The winding number of this function then gives the Chern class. Theorem 6.6 may be thought of as a similar result. The given bundle $\mathcal{V}$ is isomorphic to $T^{1,0}$ on $\mathcal{R}$ and to $T^{0,1}$ on $\mathcal{M} \backslash \overline{\mathcal{R}}$. These latter two bundles are glued together along $\Sigma$ to produce $\mathcal{V}$. Again the Chern class is realized as a winding number. The difference between these results is that here the Chern class can actually be computed from the restriction of $\mathcal{V}$ to $\Sigma$ whereas in the classical case one needs to be given, or to calculate, the trivializations over the 
hemispheres. Said somewhat differently, two generic complex line bundles in $\mathbb{C} T \mathcal{M}$ that have the same characteristic set $\Sigma$ and that agree on $\Sigma$ must be isomorphic (or conjugate). This contrasts with the fact that any two complex line bundles on $S^{2}$ are abstractly isomorphic on the equator.

Example 6.7. Consider the usual projective coordinates on the sphere, call them $z$ on $S^{2} \backslash$ ssouth pole\} and $\zeta$ on $S^{2} \backslash$ north pole\}. Then $z=\frac{1}{\zeta}$ and $z^{2} \partial_{z}=-\partial_{\zeta}$. It is easy to see that the sections

$$
\begin{aligned}
& e=\partial_{\zeta}+\partial_{\bar{z}}, \\
& f=-\partial_{\bar{\zeta}}+\partial_{z}
\end{aligned}
$$

are globally well-defined and give a trivialization of $\mathbb{C} T S^{2}$. Let $E$ and $F$ denote the trivial bundles they span. The equator

$$
\Sigma=\{z:|z|=1\}=\{\zeta:|\zeta|=1\}
$$

is the characteristic set for each of these bundles. Using polar coordinates for $z$, we see that near $\Sigma, E$ has a section

$$
\left(1-r^{2}\right) \partial_{r}+i\left(r+\frac{1}{r}\right) \partial_{\theta}
$$

and $F$ has a section

$$
\partial_{r}-\frac{i}{r}\left(\frac{1-r^{2}}{1+r^{2}}\right) \partial_{\theta}
$$

Thus $F$ is a Mizohata structure (see [14]).

To apply the theorem note that for $\mathcal{V}=F$ we have that $\mathcal{R}$ is the upper hemisphere. So we take $\tau=\partial_{z}$. We naturally use the Fubini-Study metric

$$
\left|\partial_{z}\right|=\frac{1}{1+|z|^{2}}
$$

and we let $X=\partial_{r}$. Then

$$
\beta=\frac{\langle\tau, X\rangle^{2}}{\langle X, X\rangle}=\frac{\left\langle\partial_{z}, \partial_{r}\right\rangle^{2}}{\left\langle\partial_{r}, \partial_{r}\right\rangle}=\frac{1}{8} e^{-2 i \theta}
$$

and

$$
k=\frac{1}{2 \pi i} \int_{\Sigma} \frac{d \beta}{\beta}=-2 .
$$

Thus the theorem yields

$$
\operatorname{deg}(F)=-k-\chi\left(S^{2}\right)=0,
$$

as expected since $F$ is trivial. The computation for $E$ is similar.

Proof of the Theorem. The winding number $k$ gives the algebraic number of zeroes of $\beta$ in $\mathcal{R}$. Each zero of $\beta$ in $\mathcal{R}$ occurs at a point $p$ where $\mathcal{V}_{p}=T_{p}^{1,0}$. By slightly perturbing $T^{1,0}$ we may assume that the intersection of $[\mathcal{V}]$ and $\left[T^{1,0}\right]$ is transversal. We shall see that the sign of the zero of $\beta$ is the same as the sign of the intersection. Furthermore, as we have seen, all the intersections of $[\mathcal{V}]$ and $\left[T^{1,0}\right]$ occur in $\mathcal{R}$; there are none in $\mathcal{M} \backslash \mathcal{R}$. Thus $k$ gives the algebraic number of intersections of [ $\mathcal{V}]$ and $\left[T^{1,0}\right]$ over all of $\mathcal{M}$. The formula of the theorem arises from expressing this number in terms of the integrals of the Chern classes.

We start with the sign conventions. Let $E$ be a complex line bundle on $\mathcal{M}$, and let $\sigma: \mathcal{M} \rightarrow E$ be a section with only nondegenerate zeros. Let $Z$ be the set of zeros and let $p$ be one of them. Using local coordinates in a neighborhood $U$ of $p$ and a 
trivialization of $E$ (respecting the orientation of $\mathcal{M}$ and the complex structure of $E)$ we have $\sigma(x, y)=(x, y ; f(x, y))$ with

$$
f: U \rightarrow \mathbb{C}, \quad f(0)=0, \quad \text { and } \quad d f(0) \neq 0 .
$$

The algebraic sign of the zero at $p$, denoted by $\varepsilon_{\sigma}(p)= \pm 1$, is the sign of $\operatorname{det}\left(\begin{array}{ll}a_{x} & a_{y} \\ b_{x} & b_{y}\end{array}\right)$ where $f=a+i b$.

Lemma 6.8. $\operatorname{deg}(E)=\sum_{p \in Z} \varepsilon_{\sigma}(p)$.

This is a well-known result.

Next let $\mathcal{V}$ and $\mathcal{W}$ be complex line bundles in $\mathbb{C} T \mathcal{M}$. If necessary we slightly perturb one of them in order to have that the submanifolds $[\mathcal{V}],[\mathcal{W}] \subset \mathcal{P}(\mathbb{C} T \mathcal{M})$ intersect transversally and so at only a finite number of points. We represent the orientation of $\mathcal{M}$ by a global nowhere zero 2-form $\omega \in \Lambda^{2} \mathcal{M}$. This induces orientations $\omega_{1} \in \bigwedge^{2}[\mathcal{V}], \omega_{2} \in \bigwedge^{2}[\mathcal{W}]$, and $\omega_{3} \in \bigwedge^{4} \mathcal{P}(\mathbb{C} T \mathcal{M})$.

By transversality, at each $q \in[\mathcal{V}] \cap[\mathcal{W}]$ we have

$$
\omega_{1} \wedge \omega_{2}=\lambda \omega_{3}
$$

where $\lambda$ is a nonzero real number depending on $q$. Let $\varepsilon \mathcal{V}, \mathcal{W}(p)$ be the sign of $\lambda(q)$.

\section{Definition 6.9.}

$$
\#(\mathcal{V}, \mathcal{W})=\sum \varepsilon \mathcal{V}, \mathcal{W}(p)
$$

where the summation is over all points $p \in \mathcal{M}$ with $\mathcal{V}_{p}=\mathcal{W}_{p}$.

We see that this integer is invariant under deformations of the bundles $\mathcal{V}$ and $\mathcal{W}$, and so is also independent of any original perturbation, and that $\#(\mathcal{V}, \mathcal{W})=$ $\#(\mathcal{W}, \mathcal{V})$

To compute $\varepsilon \mathcal{V}, \mathcal{W}\left(p_{0}\right)$ choose a section $Z$ of $\mathcal{W}$ near $p_{0}$ of the form $Z=\partial_{x}+f \partial_{y}$ with respect to some oriented coordinates $(x, y)$ and let $Z^{\prime}$ be a section of $\mathbb{C} T \mathcal{M}$ near $p$ independent of $Z$. Then $\mathcal{V}$ has a section of the form $X=Z+h Z^{\prime}$ with $h=a+\sqrt{-1} b$ vanishing only at $p_{0}$. With $Z, Z^{\prime}$ we trivialize $\mathcal{P}(\mathbb{C} T \mathcal{M})$ on a neighborhood $U$ of $p_{0}$ as $U \times \mathbb{C P}^{1}$ by mapping $\zeta Z+\zeta^{\prime} Z^{\prime}$ over $p$ to $\left(p,\left[\zeta, \zeta^{\prime}\right]\right)$. In this trivialization $[\mathcal{W}]$ is the set of points of the form $(p ;[1,0])$. Near $p_{0}$ the section $[\mathcal{V}]$ is $p \mapsto[1, h(p)]$. From this one sees at once that if $h=a+\sqrt{-1} b$, then $\varepsilon_{\mathcal{V}, \mathcal{W}}(p)$ is the sign of the zero of $a+i b$ at $p_{0}$,

$$
\varepsilon_{\mathcal{V}, \mathcal{W}}\left(p_{0}\right)=\operatorname{sign} \operatorname{det}\left(\begin{array}{ll}
a_{x} & a_{y} \\
b_{x} & b_{y}
\end{array}\right) .
$$

In particular, if $\mathcal{W}=T^{1,0}$, we may use

$$
Z=\partial_{z}, \quad Z^{\prime}=\partial_{\bar{z}} .
$$

If $\mathcal{V}$ intersects $T^{1,0}$ transversally at $p_{0}$, then it has a section $X=\partial_{z}+(a+i b) \partial_{\bar{z}}$ and $\varepsilon_{\mathcal{V}, T^{1,0}}(p)$ is the sign of the zero of $a+\sqrt{-1} b$. Also, with $\tau=\alpha \partial_{z}$,

$$
\begin{aligned}
\beta & =\frac{1}{|\zeta|^{2}}\langle\tau, \zeta\rangle\langle\tau, \bar{\zeta}\rangle \\
& =\frac{1}{|\zeta|^{2}}\left\langle\alpha \partial_{z}, \zeta\right\rangle\left\langle\alpha \partial_{z}, \bar{\zeta}\right\rangle \\
& =\frac{\alpha^{2}}{|\zeta|^{2}}(a+i b) .
\end{aligned}
$$

So the sign of the zero of $\beta$ agrees with the sign of the intersection; thus,

$$
k=\#\left(\mathcal{V}, T^{1,0}\right) .
$$


We now relate this number to the Chern classes.

Lemma 6.10. $\operatorname{deg}(\mathcal{V})+\operatorname{deg}(\mathcal{W})=-\#(\mathcal{V}, \mathcal{W})$

Proof. Let $\sigma_{\mathcal{V}}: \mathcal{M} \rightarrow \mathcal{V}$ be a section and perturb it if necessary to have $\sigma_{\mathcal{V}} \neq 0$ at points of $\mathcal{V} \cap \mathcal{W}$. Let $\pi: \mathbb{C} T \mathcal{M} \rightarrow \mathcal{W}^{\perp}$ be projection along $\mathcal{W}$. Consider the section of $\mathcal{W}^{\perp}$ given by $\sigma=\pi \sigma_{\mathcal{V}}$. We have that $\sigma(q)=0$ implies that either $\sigma_{\mathcal{V}}(q)=0$ or $\sigma_{\mathcal{V}}(q) \in \mathcal{W}$ but not both. It is a simple computation to see that if $\sigma_{\mathcal{V}}(q)=0$, then the zero of $\sigma$ at $q$ has the same sign as that of $\sigma_{\mathcal{V}}$ at $q$ and that if $\mathcal{V}$ and $\mathcal{W}$ intersect at $q$, then the zero of $\sigma$ has the same sign as the intersection. Thus

$$
\begin{aligned}
\operatorname{deg}\left(\mathcal{W}^{\perp}\right) & =\sum_{p \in Z_{\sigma}} \varepsilon_{\sigma}(p) \\
& =\sum_{p \in Z_{\sigma \mathcal{V}}} \varepsilon_{\sigma \mathcal{V}}(p)+\#\{\mathcal{V} \cap \mathcal{W}\}
\end{aligned}
$$

So

$$
-\operatorname{deg}(\mathcal{W})=\operatorname{deg}(\mathcal{V})+\#\{\mathcal{V} \cap \mathcal{W}\}
$$

We apply this lemma with $\mathcal{W}=T^{1,0}$ and use $k=\#\left(\mathcal{V}, T^{1,0}\right)$ and $\operatorname{deg}\left(T^{1,0}\right)=$ $\chi(\mathcal{M})$ to obtain the theorem.

\section{HyPO-COMPLEX STRUCTURES}

The fact that $B_{\mathbb{R}}$ is a separating hypersurface for $\mathcal{P}(\mathbb{C} T \mathcal{M})$ provides a simple proof of an interesting result about global partial differential equations. Again $\mathcal{M}$ is an orientable two-dimensional manifold, and $\mathcal{V}$ is a complex line bundle in $\mathbb{C} T \mathcal{M}$.

Definition 7.1. A distribution $f$ is a solution for $\mathcal{V}$ over an open set $U$ if $L f=0$ for every section $L$ of $\mathcal{V}$ over $U$.

Definition 7.2. $(\mathcal{M}, \mathcal{V})$ is a hypo-complex structure if for each $p \in \mathcal{M}$ there exists some open neighborhood $U$ and some $C^{\infty}$ solution $Z$ over $U$ such that any solution in some neighborhood of $p$ is a holomorphic function of $Z$ near $p$.

The name reflects the fact that this class of structures is modeled on, but does not coincide with, the complex structures. Here is the result we seek to prove.

Theorem 7.3. Let $(\mathcal{M}, \mathcal{V})$ be a hypo-complex structure. There exists a smooth family of sub-bundles $\mathcal{V}^{t}, 0 \leq t \leq 1$, that are complex structures for $0 \leq t<1$ and with $\mathcal{V}^{1}=\mathcal{V}$.

Lemma 7.4. $(\mathcal{M}, \mathcal{V})$ is a complex manifold if for each $p \in \mathcal{M}$ there exists a vector $L \in \mathcal{V}_{p}$ such that $\Re L$ and $\Im L$ are linearly independent.

Proof. This is essentially the existence of isothermal coordinates (see, for instance, [8]).

Remark 7.5. Fix an orientation for $\mathcal{M}$. If the ordered set $\{\Re L, \Im L\}$ agrees with this orientation, then so does the orientation of the complex structure that it defines.

Here is the corresponding result for hypo-complex structures. The proof, in coordinate-free form, may be found in [30. Theorem III.6.2]. 
Lemma 7.6. $(\mathcal{M}, \mathcal{V})$ is a hypo-complex structure if and only if for each $p \in \mathcal{M}$ there exists a smooth section $L$ of $\mathcal{V}$ in a neighborhood of $p$ and coordinates $(x, y)$ such that

$$
\begin{aligned}
& L=\partial_{x}+i b(x, y) \partial_{y}, \\
& b \geq 0, \\
& b(x, 0) \text { not identically zero in any neighborhood of } x=0 .
\end{aligned}
$$

Now fix an orientation for $\mathcal{M}$. We say that $\mathcal{V}$ is positively oriented at $p$ if $\partial_{x} \wedge \partial_{y}$ gives the orientation of $\mathcal{M}$; otherwise, $\mathcal{V}$ is negatively oriented at $p$. This makes sense because if $\tilde{L}$ is also a section of $\mathcal{V}$ and $(\tilde{x}, \tilde{y})$ are also local coordinates near $p$ for which

$$
\begin{aligned}
\tilde{L} & =\partial_{\tilde{x}}+i \tilde{b}(\tilde{x}, \tilde{y}) \partial_{\tilde{y}}, \\
\tilde{b} & \geq 0,
\end{aligned}
$$

then $\partial_{\tilde{x}} \wedge \partial_{\tilde{y}}=\lambda \partial_{x} \wedge \partial_{y}$ with $\lambda>0$. Similarly, it is easy to see that if $\mathcal{V}$ is positively oriented at one point of $\mathcal{M}$, then it is positively oriented at all points of $\mathcal{M}$.

Proof of Theorem 7.3. We relate the above lemma to our decomposition

$$
\mathcal{P}(\mathbb{C} T \mathcal{M})=B_{+} \cup B_{\mathbb{R}} \cup B_{-} .
$$

First, let $\mathcal{V}$ be any complex line sub-bundle of $\mathbb{C} T \mathcal{M}$. Fix some point $p \in \mathcal{M}$ and choose local coordinates and a local section $L$ of $\mathcal{V}$ such that

(1) $\left\{\partial_{x}, \partial_{y}\right\}$ gives the orientation of $M$;

(2) $L=\partial_{x}+i b(x, y) \partial_{y}$.

If at some point $q$ near $p, b(q)=0$, then $\left[\mathcal{V}_{q}\right] \in B_{\mathbb{R}}$. If $b(q)>0$, then near $q, L$ defines a complex structure with the same orientation as $\mathcal{M}$ and so $\left[\mathcal{V}_{q}\right] \in B_{+}$. In the remaining case, $\left[\mathcal{V}_{q}\right] \in B_{-}$.

Now let $\mathcal{V}$ be hypo-complex. Then $[\mathcal{V}]$ is a section of $B_{+} \cup B_{\mathbb{R}}$ when $\mathcal{V}$ is positively oriented and a section $B_{-} \cup B_{\mathbb{R}}$ when $\mathcal{V}$ is negatively oriented. We limit ourselves, without loss of generality, to the positive case. Clearly, there is a fiber-preserving deformation

$$
\phi_{t}: B_{+} \cup B_{\mathbb{R}} \rightarrow B_{+} \cup B_{\mathbb{R}}
$$

with $\phi_{1}$ the identity and $\phi_{t}\left(B_{+} \cup B_{\mathbb{R}}\right) \subset B_{+}$for $t<1$. So, consider the family of bundles, $\mathcal{V}^{t}$, defined by

$$
\left[\mathcal{V}^{t}\right]_{x}=\phi_{t}\left([\mathcal{V}]_{x}\right)
$$

We have $\mathcal{V}^{1}=\mathcal{V}$ while each $\mathcal{V}^{t}$, for $t<1$, defines a complex structure on $\mathcal{M}$.

Note we have not used the condition that $b(x, 0)$ is nowhere identically zero. For example, a real nowhere zero vector field $X$ on a torus is clearly the limit of the complex structures given by $X+i \epsilon Y$, where $Y$ is orthogonal to $X$.

Corollary 7.7. Let $\mathcal{V}$ be a hypo-complex structure on a compact, orientable, twodimensional manifold $\mathcal{M}$. Then $\operatorname{deg}(\mathcal{V})= \pm \chi(\mathcal{M})$. 


\section{Hypo-AnAlytiC StruCtures}

Let $\mathcal{M}^{n}$ be a smooth manifold, $\mathcal{V} \subset \mathbb{C} T \mathcal{M}$ a sub-bundle of rank $r$. A condition on $\mathcal{V}$ finer than involutivity is that of local integrability (see [1], [30]): $\mathcal{V}$ is locally integrable if for each $p \in \mathcal{M}$ there is a neighborhood $U$ of $p$ and $Z^{1}, \ldots, Z^{n-r}$ : $U \rightarrow \mathbb{C}$ smooth with $d Z^{1}, \ldots, d Z^{n-r}$ independent such that $L Z^{i}=0$ for all $i$ and for each section $L$ of $\mathcal{V}$ over $U$.

An even finer condition is that $\mathcal{V}$ admits a hypo-analytic structure. A hypoanalytic structure on $\mathcal{M}$ (see [2], [30, Definition III.1.1]) is the datum of a family of functions

$$
\mathfrak{F}=\left\{Z_{\alpha}: U_{\alpha} \subset \mathcal{M} \rightarrow \mathbb{C}^{n-r}: \alpha \in A, U_{\alpha} \text { open and connected, } Z_{\alpha} \text { smooth }\right\}
$$

such that

(1) $\mathcal{M}=\bigcup_{\alpha \in A} U_{\alpha}$,

(2) for all $\alpha \in A, d Z_{\alpha}^{1}, \ldots, d Z_{\alpha}^{n-r}$ are independent on $U_{\alpha}$, and

(3) for all $\alpha, \beta \in A$, if $p \in U_{\alpha} \cap U_{\beta}$, then there is $h_{\alpha \beta}$ holomorphic defined in a neighborhood of $Z_{\beta}(p)$ such that $Z_{\alpha}=h_{\alpha \beta} \circ Z_{\beta}$.

A hypo-analytic structure always determines a locally integrable sub-bundle $\mathcal{V}$ of $\mathbb{C} T \mathcal{M}: v \in \mathcal{V}$ if and only if $\left\langle d Z_{\alpha}^{i}, v\right\rangle=0$ for all $\alpha$ and $i . \mathcal{V}$ is said to admit a hypoanalytic structure if it arises in this manner, and is referred to as the structure bundle of the hypo-analytic structure. It is not known whether a locally integrable sub-bundle always admits a hypo-analytic structure. However,

Proposition 8.1. Let $\mathcal{M}$ be a compact orientable manifold. Let $E \rightarrow \mathcal{M}$ be a line bundle. Then there is a hypo-analytic structure on $\mathcal{M}$ whose structure bundle is isomorphic to E.

Proof. We may assume that $\mathcal{M}$ is a real analytic manifold and $E$ a real analytic bundle. Then $E$ can be realized as a real analytic sub-bundle of $\mathbb{C} T \mathcal{M}$. Now apply Theorem III.1.1 of [30].

We will give a more explicit proof in the two-dimensional case. The line bundle can be realized as a sub-bundle of $\mathcal{V} \subset \mathbb{C} T \mathcal{M}$ such that the induced section $[\mathcal{V}]$ : $\mathcal{M} \rightarrow \mathcal{P}(\mathbb{C} T \mathcal{M})$ has transverse intersection with $B_{\mathbb{R}}$. The intersection occurs over a finite union $\Sigma$ of closed curves; these are composed of the characteristic points of $\mathcal{V}$. Let $\gamma_{j}$ be one of those curves. It has a neighborhood $U$ diffeomorphic to an annulus $\{z \in \mathbb{C}: 1 / R<|z|<R\}, R>1$, with $\gamma_{j}$ mapped onto $|z|=1$. Over such a neighborhood $\mathcal{V}$ is trivial. So $\mathcal{V}$ has a nonvanishing section

$$
\alpha \partial_{z}+\beta \partial_{\bar{z}}
$$

over $U$. The condition that $\mathcal{V}$ is real over $|z|=1$ is equivalent to $|\alpha / \beta|=1$ when $|z|=1$, and the transversality condition implies that $d|\alpha / \beta| \neq 0$ when $|\alpha / \beta|=1$. So in a neighborhood of $|z|=1$ we have $|\alpha(z) / \beta(z)|=1$ if and only if $|z|=1$. Since neither $\alpha$ nor $\beta$ vanishes on the characteristic points, we may assume that $\alpha$ never vanishes on $U$. Dividing by $\alpha$, we may thus assume that in (8.2), $\alpha=1$. Since $\beta \neq 0$ on $U, \beta=\rho e^{\sqrt{-1} \psi}$ for some real-valued functions $\psi, \rho$ with $\rho=1$ and $d \rho \neq 0$ when $|z|=1$. Let $m$ be the winding number of $e^{\sqrt{-1} \psi}$. If $m \neq 0$ then, depending on the sign of $\rho-1$ on $|z|>1$ and the sign of $m, \beta$ is homotopic to either $z^{m}$ or $(\bar{z})^{-m}$ through functions of the form $\rho_{t} e^{\sqrt{-1} \psi_{t}}$ with $\psi_{t}, \rho_{t}$ smooth, real-valued, $d \rho_{t} \neq 0$, and with $\rho_{t}=1$ if and only if $|z|=1$. If $m=0$, then again depending on the 
sign of $\rho-1$ on $|z|>1, \beta$ is homotopic to $|z|^{4}$ or $1 /|z|^{4}$ (the power 4 is chosen for convenience). These deformations can be taken so as to leave $\beta$ unchanged near the boundary of $U$. Thus $\mathcal{V}$ can be deformed through line sub-bundles of $\mathbb{C} T \mathcal{M}$ keeping the characteristic set fixed and the condition of transversality valid throughout the deformation, to one which in suitable neighborhoods $U_{\gamma_{j}}$ of the components $\gamma_{j}$ of the characteristic set, with suitable coordinates $(x, y)$, has a section

$$
\begin{array}{llll}
\partial_{z}+z^{m} \partial_{\bar{z}} & \text { or } & \partial_{z}+(\bar{z})^{-m} \partial_{\bar{z}} & \text { if } m \neq 0, \\
\partial_{z}+|z|^{4} \partial_{\bar{z}} & \text { or } & \partial_{z}+|z|^{-4} \partial_{\bar{z}} & \text { if } m=0 .
\end{array}
$$

We now choose a hypo-analytic structure. For each $p \in \mathcal{M} \backslash \Sigma$, choose a small coordinate ball $U_{p} \subset \mathcal{M} \backslash \Sigma$ centered at $p$ and a solution $Z_{p}: U_{p} \rightarrow \mathbb{C}$ (Definition 7.1). These solutions give a hypo-analytic (in fact hypo-complex) structure on $\mathcal{M} \backslash \Sigma$. The existence of a hypo-analytic structure on $\mathcal{M}$ will be completed by presenting a global solution for each of the vector fields (8.3) in a suitable neighborhood of the circle $\gamma$. If $\mathcal{V}$ is spanned by $\partial_{z}+z^{m} \partial_{\bar{z}}$ along $\gamma \subset \Sigma(m \neq 0)$, take $Z_{\gamma}=\frac{1}{m+1} z^{m+1}-\bar{z}$ if $m \neq-1$, and $Z_{\gamma}=z e^{-\bar{z}}$ if $m=-1$, and if $\mathcal{V}$ is spanned by $\partial_{z}+|z|^{4} \partial_{\bar{z}}$, take $Z_{\gamma}=z^{3} / 3+1 / \bar{z}$. Likewise one has global solutions in the remaining cases. On $U_{\gamma} \cap U_{p}, Z_{\gamma}$ is related to the solution $Z_{p}$ by a holomorphic function, since there the structure is elliptic. Thus the functions $Z_{p}$ together with these $Z_{\gamma}$ give a hypo-analytic structure.

The number $k_{j}$ associated through (6.5) with the vector fields in (8.3) are, for those in the first line, $|m|$ and $-|m|$, respectively, and 0 for those in the second.

\section{REFERENCES}

[1] Andreotti, A. and Hill, C. D., Complex characteristic coordinates and tangential CauchyRiemann equations, Ann. Scuola Norm. Sup. Pisa, Sci. Fis. Mat. 26 (1972), 299-324. MR 57:717

[2] Baouendi, M. S., Chang, C. H., and Treves, F., Microlocal hypoanalyticity and extension of CR functions, J. Differential Geom. 18 (1983), 331-391. MR 85h:32030

[3] Bott, R., On a topological obstruction to integrability, in: Global Analysis, Proc. Sympos. Pure Math. 16, Amer. Math. Soc. (1970), 127-131. MR 42:1155

[4] Bott, R. and Tu, L. W., Differential forms in algebraic topology, Springer-Verlag, Berlin, Heidelberg, New York, 1982. MR 83i:57016

[5] Brunella, M. On transversely holomorphic flows. I, Invent. Math. 126 (1996), 265-279. MR 97j:58121

[6] Cadek, M. and Vanžura, J., On complex structures in 8-dimensional vector bundles, Manuscripta Math. 95 (1998), 323-330. MR 98m:57034

[7] - On 2-distributions in 8-dimensional vector bundles over 8-complexes, Colloq. Math. 70 (1996), 25-40. MR 96k:57021

[8] Chern, S. S., An elementary proof of the existence of isothermal parameters on a surface, Proc. Amer. Math. Soc. 6 (1955), 771-782. MR 17:657g

[9] Ghys, É., On transversely holomorphic flows. II, Invent. Math. 126 (1996), 281-286. MR 97j:58122

[10] Heaps, T., Almost complex structures on eight and ten-dimensional manifolds, Topology 9 (1970), 111-119. MR 41:9283

[11] Hirzebruch, F. and Hopf, H., Felder von Flächenelementen in 4-dimensionalen Mannigfeltigkeiten, Math. Ann. 136 (1958), 156-172. MR 20:7272

[12] Hsiung, C., Almost complex structures and complex structures, Series in Pure Mathematics, Vol. 20, World Scientific Publishing Co., Singapore, 1995. MR 96j:53033

[13] Husemoller, D., Fibre Bundles, third edition, Springer-Verlag, Berlin, Heidelberg, New York, 1993. MR 94k:55001

[14] Jacobowitz, H., Global Mizohata Structures, J. Geom. Anal. 3 (1993), 153-193. MR 94c:58190 
[15] _ Transversely holomorphic foliations and CR structures, VI Workshop on Partial Differential Equations, Part I (Rio de Janeiro, 1999), Mat. Contemp. 18 (2000), 175-194. MR 2002f:32061

[16] Kervaire, M., A note on obstructions and characteristic classes, Amer. J. Math. 81 (1959), 773-784. MR 21:6585

[17] MacPherson, R., Singularities of vector bundle maps, Proceedings of Liverpool Singularities Symposium, I (1969/70), pp. 316-318. Lecture Notes in Math., Vol. 192, Springer-Verlag, Berlin, 1971. MR 43:4055

[18] _ Generic vector bundle maps. Dynamical Systems, Proc. Sympos., Univ. Bahia, Salvador, 1971, pp. 165-175, Academic Press, New York, 1973. MR 49:3962

[19] Mello, M. H. de P. L., Two-plane sub-bundles of nonorientable real vector-bundles, Manuscripta Math. 57 (1987), 263-280. MR 88c:55020

[20] Massey, W. S. On the Stiefel-Whitney classes of a manifold, Amer. J. Math. 82 (1960), 92-102. MR 22:1918

[21] Mendoza, G. A., Elliptic structures, Multidimensional complex analysis and partial differential equations (São Carlos, 1995), Contemp. Math. 205 (1997), 219-234. MR 98c:32041

[22] Milnor, J. and Stasheff, J., Characteristic Classes, Princeton University Press, 1974. MR 55:13428

[23] Peterson, F., Some remarks on Chern classes, Ann. Math. 69 (1959), 414-420. MR 21:1593

[24] Shulman, H., Secondary obstructions to foliations, Topology 13 (1974), 177-183. MR 49:9855

[25] Steenrod, N., Topology of fiber bundles, Princeton University Press, 1951 (ninth printing, 1974). MR 12:522b

[26] Thomas, E., Fields of tangent 2-planes on even dimensional manifolds, Ann. Math. (2) 86 (1967), 349-361. MR 35:3699

[27] , Fields of tangent k-planes on manifolds, Invent. Math. 3 (1967), 334-347. MR 36:903

[28] Complex Structures on Real Vector Bundles, Amer J. Math. 89 (1967), 887-908. MR 36:3375

[29] — Secondary obstructions to integrability. Dynamical systems (Proc. Sympos., Univ. Bahia, Salvador, 1971), pp. 655-661, Academic Press, New York, 1973. MR 49:8035

[30] Treves, F., Hypo-analytic Structures: Local Theory, Princeton University Press, 1992. MR 94e:35014

[31] Turaev, V., Torsion invariants of Spin $^{c}$-structures on 3-manifolds, Math. Res. Lett. 4 (1997), 679-695. MR 98k:57038

[32] Wood, J. W., Foliations on 3-manifolds, Ann. Math. (2) 89 (1969), 336-358. MR 40:2123

Department of Mathematics, Rutgers University, Camden, New Jersey 08102

E-mail address: jacobowi@camden.rutgers.edu

Department of Mathematics, Temple University, Philadelphia, Pennsylvania 19122

E-mail address: gmendoza@math.temple.edu 